\title{
Lernen, Lehren und Forschen neu denken: Eine Agenda für die Hochschule der Zukunft
}

Die Hochschule der Zukunft wird vor allem eines: Sie wird mehr auf die Diversität zukünftiger Zielgruppen von Studierenden eingehen müssen. Und sie wird digitaler werden. Unterschiedlicher in ihren Strukturen und unterschiedlicher in den damit verbundenen Lernszenarien der Studierenden, Lehrszenarien der Professorinnen, Professoren und der Lehrenden - und letztlich auch in ihren Forschungsansätzen. Das Selbstverständnis der Hochschulen wandelt sich - weiter! Im Abschlusskapitel des Buches haben wir vier Szenarien für Profile der zukünftigen Hochschule herausgearbeitet, die auf Daten des internationalen NextSkills Delphi basieren. In diesem Kapitel geht es aber um die Binnenfragen der Hochschulentwicklung: Lehren, Lernen und Forschen der Zukunft und die Frage, wie sich das Studium entwickelt?

Wie sieht also eine Agenda für die Hochschule der Zukunft aus? Die Beschäftigung mit diesem Thema führt unweigerlich dazu, sich das neue Lehren und Lernen auszumalen; die Art und Weise, wie in Zukunft studiert wird. Dabei stehen einerseits pädagogische Aspekte des Lehrens und Lernens im Vordergrund, wie beispielsweise die Weiterentwicklung von Prüfungs- und Bewertungspraktiken, Peer-Learning und Peer-Validierungs-Ansätze, das Umsetzen akademischen Lernens und Lehrens als Lerngemeinschaft sowie ein verstärkter Fokus auf Future Skills. Neben diesen eher pädagogischen und studierbezogenen Aspekten gibt es aber weitere.

Eine Agenda für die Hochschule der Zukunft muss auch die Struktur der Hochschule, die innere Gliederung sowie die Organisation des Studiums in den Blick nehmen. Was ändert sich strukturell? Hinsichtlich der Organisation des Studiums werden Hochschulen sich grundlegend wandeln müssen. Mehr Studierende, neue Zielgruppen und eine vorher nicht dagewesene Diversität der Zielgruppen, die es zu wertschätzen und in personalisierten Studiensituationen zu betreuen gilt, kommen auf die Hochschulen zu. Dazu wird die Funktion der Hochschulen in Bezug auf gesellschaftliche Integration und die soziale Dimension des Studierens in einer akademischen Bildungsgesellschaft immer bedeutsamer. Damit verbunden gewinnt das Konzept des lebenslangen Lernens für Hochschulen an Bedeutung und alle 
damit zusammenhängenden Veränderungen, die wie bei einem Dominosteinspiel in der Hochschullehre und -organisation betroffen sind, wenn man diesen Stein anstößt. Da ist beispielsweise das Konzept der Microcredentials, der alternativen Zertifizierungssysteme, die es Lernenden ermöglichen, das eigene Portfolio der Qualifikationen und Kompetenzen digital und in größerer Selbstbestimmung zu organisieren und von Hochschulen fordern, die Systeme für Anerkennung und Anrechnung zu professionalisieren. Digitalisierung ermöglicht Flexibilisierung von Raum- und Zeitstrukturen und eine größere Transparenz aller studienbezogenen Informationssysteme über den gesamten Studien-Lifecycle. In einer digitalen Welt erleben wir eine abnehmende Bedeutung von Wissensvermittlung und einen steigenden Bedarf an Begleitung, Betreuung und Coaching in einer vielfältigeren Studienwelt. Darüber hinaus spielt die Entkoppelung von Prozessen des Lehrens, des Prüfens und der Zertifizierung von Kompetenzen eine zunehmend große Rolle. Dabei ist es bemerkenswert, dass die Expertinnen und Experten im internationalen NextSkills Delphi erwarten, dass die organisationalen und strukturellen Veränderungen erst wesentlich später an Relevanz gewinnen werden als die Wandelprozesse, die sich auf akademische Lehr-/ Lerndesigns beziehen.

Legt man die geänderten Rahmenbedingungen in einer Bildungsgesellschaft zugrunde und den Änderungsdruck, der auf akademische Qualifizierungsprozesse wirkt, so ergeben sich für Hochschulen neue Anforderungen an ein modernes, weiterentwickeltes Hochschulmodel. Die folgenden Aspekte zeigen den Entwicklungskorridor auf, in dem Hochschulen sich derzeit befinden. Die Hochschule der Zukunft wird ihre Profilpunkte entlang dieser Agenda ausrichten müssen.

Im Folgenden werden alle diejenigen Konzepte beschrieben, die sich im NextSkills Projekt als bedeutsam erwiesen haben. ${ }^{39}$ Sie sind aufgeteilt in drei Kapitel und beginnen mit einem Gedankenexperiment, in dem die Entwicklung der Hochschule skizziert wird (Kapitel C2.1). Darauf folgt ein Überblick zum Thema Lehre und Lernen (Kapitel C2.2), zur Organisation und den Strukturen von der Hochschule der Zukunft (Kapitel C2.3) sowie ein zusammenfassendes Fazit (Kapitel C2.4).

39 Die im Folgenden beschriebenen Konzeptionen sind das zusammengefasste Ergebnis einer Analyse aus dem NextSkills Projekt (www.NextSkills.org). Mitberücksichtigt sind dabei die über 100 Konzeptionen zum Curriculum 4.0, mit denen sich Hochschulen auf die Ausschreibung des Programms „Curriculum 4.0“ beworben haben. Dieses wurde 2017 von der Carl-Zeiss-Stiftung und dem Stifterverband mit dem Ziel ins Leben gerufen, curriculare Reformprojekte auszuzeichnen, die neue Lösungsansätze im Umgang mit digitalen Medien aufzeigen. 


\section{C2.1 Hochschule der Zukunft: Ein Gedankenexperiment}

Wenn man das heutige Hochschulmodell in eine postmoderne Zukunft transponiert - welche Strukturen gewinnen dann an Bedeutung? Legt man die geänderten Rahmenbedingungen in einer Bildungsgesellschaft zugrunde und den Änderungsdruck, der auf akademische Qualifizierungsprozesse wirkt, so ergeben sich auch für Hochschulen neue Anforderungen an ein modernes, weiterentwickeltes Hochschulmodell. Die folgenden Aspekte (Tabelle 3) sind das Ergebnis eines Gedankenexperiments und zeigen den Entwicklungskorridor auf, in dem Hochschulen sich derzeit befinden. Die Hochschule der Zukunft wird sich entlang dieser Profilpunkte ausrichten müssen.

Tab. 3 Eckpunkte der Hochschule der Zukunft

\begin{tabular}{|c|c|c|}
\hline Dimension & $\begin{array}{l}\text { derzeitiges } \\
\text { Hochschulmodell }\end{array}$ & $\begin{array}{l}\text { zukünftiges } \\
\text { Hochschulmodell (postmodern) }\end{array}$ \\
\hline & \multicolumn{2}{|c|}{ von ... (möglicher Entwicklungspfad) ...zu } \\
\hline Abschlüsse & $\begin{array}{l}\text { Ziel ist das Erreichen eines klar } \\
\text { definierten Gesamtabschlusses für } \\
\text { das Studium, dabei werden die Ab- } \\
\text { schlussbezeichnungen hoheitlich von } \\
\text { der Hochschule vergeben. }\end{array}$ & $\begin{array}{l}\text { Das Studium setzt sich aus kleinen } \\
\text { Studieneinheiten zusammen, die } \\
\text { auch von unterschiedlichen (Hoch- } \\
\text { schul-)Anbietern kommen können. } \\
\text { Es wird mehr Kurzformate, Zertifi- } \\
\text { katskurse, Kontaktstudienmöglich- } \\
\text { keiten und Short-Courses geben. } \\
\text { Daraus entstehen Patchwork-Studi- } \\
\text { enverläufe, die dann zu größe- } \\
\text { ren Abschlusszertifikaten, wie } \\
\text { beispielsweise einem Studienab- } \\
\text { schluss, zusammengefügt und } \\
\text { von einer Hochschule zertifiziert } \\
\text { werden können. }\end{array}$ \\
\hline $\begin{array}{l}\text { Anerkennung vor- } \\
\text { heriger Kenntnisse } \\
\text { \& Erfahrungen }\end{array}$ & $\begin{array}{l}\text { Anerkennung ist möglich, aber es } \\
\text { gibt wenig tatsächliche Anerken- } \\
\text { nungspraxis. }\end{array}$ & $\begin{array}{l}\text { Viel Anerkennungspraxis, Hoch- } \\
\text { schulen entwickeln professionelle } \\
\text { Prozesse für Kompetenzdiagnose } \\
\text { und die Anerkennung von Vorleis- } \\
\text { tungen und Erfahrungen. }\end{array}$ \\
\hline Zertifizierung & $\begin{array}{l}\text { Lehre/ Vermittlung (Tutoring, } \\
\text { Lehrveranstaltungen), Prüfung und } \\
\text { Zertifizierung sind im Rahmen einer } \\
\text { Institution gekoppelt. }\end{array}$ & $\begin{array}{l}\text { Lehre/ Vermittlung (Tutoring, } \\
\text { Lehrveranstaltungen), Prüfungen } \\
\text { und Zertifizierung (Abschlussprü- } \\
\text { fung) sind entkoppelt und können } \\
\text { von verschiedenen Institutionen } \\
\text { durchgeführt werden. }\end{array}$ \\
\hline
\end{tabular}




\begin{tabular}{|c|c|c|}
\hline Dimension & $\begin{array}{l}\text { derzeitiges } \\
\text { Hochschulmodell }\end{array}$ & $\begin{array}{l}\text { zukünftiges } \\
\text { Hochschulmodell (postmodern) }\end{array}$ \\
\hline $\begin{array}{l}\text { Studienpfad/ } \\
\text { Taktung }\end{array}$ & $\begin{array}{l}\text { Studienverlauf ist durch Modul- und } \\
\text { Prüfungsplan in der Studienordnung } \\
\text { klar und nur mit geringer Flexibilität } \\
\text { vorgegeben. } \\
\text { Studium ist anhand von Zeiteinhei- } \\
\text { ten strukturiert (ECTS). } \\
\text { Klare Unterscheidung von Teilzeit } \\
\text { und Vollzeitstruktur. }\end{array}$ & $\begin{array}{l}\text { Studienverlauf ist flexibel und } \\
\text { durch große Wahlbereiche be- } \\
\text { stimmt. } \\
\text { Studium ist anhand von inhaltli- } \\
\text { chen Kriterien strukturiert. } \\
\text { Flexiblere, individuelle Zeitstruk- } \\
\text { tur } \\
\text { mehr berufs- und lebensbegleiten- } \\
\text { de Modelle. }\end{array}$ \\
\hline \multirow[t]{3}{*}{ Curriculum } & $\begin{array}{l}\text { Im Studium sind klar definierte } \\
\text { Qualifikationsziele vorgegeben, die } \\
\text { für alle Studierenden gleichermaßen } \\
\text { gelten und aus denen die Inhalte und } \\
\text { Methoden der Module im Studien- } \\
\text { verlauf abgeleitet werden. } \\
\text { Berufsprofile werden als normati- } \\
\text { ves Paradigma für Studieninhalte } \\
\text { herangezogen. }\end{array}$ & $\begin{array}{l}\text { Studieninhalt ist zunehmend } \\
\text { orientiert an langfristiger Beschäf- } \\
\text { tigungsfähigkeit und an individuel- } \\
\text { len Bildungszielen, Interessen und } \\
\text { Bedürfnissen. } \\
\text { Im Vordergrund stehen mehr } \\
\text { grundlegende Handlungskom- } \\
\text { petenzen und die Befähigung } \\
\text { zum Umgang mit übergreifenden } \\
\text { Fähigkeiten. }\end{array}$ \\
\hline & $\begin{array}{l}\text { Ein Methoden- und Inhaltskanon } \\
\text { ist an Fakultäten und Disziplinen } \\
\text { orientiert. }\end{array}$ & $\begin{array}{l}\text { Das Curriculum ist an zentralen } \\
\text { Problemstellungen eines Praxisfel- } \\
\text { des orientiert } \\
\text { Die Problemorientierung bedingt } \\
\text { eine stärker interdisziplinäre } \\
\text { Ausrichtung }\end{array}$ \\
\hline & $\begin{array}{l}\text { Wenig digitaler Import von Curri- } \\
\text { cula }\end{array}$ & $\begin{array}{l}\text { Viel digitale Kooperation und } \\
\text { digitaler Im- und Export zwischen } \\
\text { akademischen Institutionen }\end{array}$ \\
\hline $\begin{array}{l}\text { Wissenschafts-/ } \\
\text { Hochschulstruktur }\end{array}$ & $\begin{array}{l}\text { Hochschulen sind in disziplinäre } \\
\text { Einheiten, die Fakultäten struktu- } \\
\text { riert; sie sind inhaltlich maßgebend } \\
\text { und für das Studium strukturgebend. }\end{array}$ & $\begin{array}{l}\text { Hochschulen sind stärker durch } \\
\text { interdisziplinäre/ transdisziplinäre } \\
\text { Kooperationsformen organisiert } \\
\text { Das Studium ist stärker anhand } \\
\text { von übergreifenden Fragestel- } \\
\text { lungen und interdisziplinären/ } \\
\text { transdisziplinären Arbeitseinheiten } \\
\text { organisiert. }\end{array}$ \\
\hline
\end{tabular}




\begin{tabular}{|c|c|c|}
\hline Dimension & $\begin{array}{l}\text { derzeitiges } \\
\text { Hochschulmodell }\end{array}$ & $\begin{array}{l}\text { zukünftiges } \\
\text { Hochschulmodell (postmodern) }\end{array}$ \\
\hline \multirow[t]{2}{*}{ Lernmodell } & $\begin{array}{l}\text { Lernen folgt prinzipiell der Vorstel- } \\
\text { lung eines Wissensgefälles, welches } \\
\text { es auszugleichen gilt. } \\
\text { Die Lehre ist expert/innenorientiert } \\
\text { Professor/innen organisieren Wis- } \\
\text { senstransfer }\end{array}$ & $\begin{array}{l}\text { Lernen folgt der Vorstellung, dass } \\
\text { Studierende und Lehrende eine } \\
\text { Lerngemeinschaft bilden (Renais- } \\
\text { sance des Ideals der Universitas) }\end{array}$ \\
\hline & $\begin{array}{l}\text { Prüfungsorientiertes Lernen: Lernen } \\
\text { ist auf Prüfungen ausgerichtet. } \\
\text { Studium folgt der Vorstellung, dass } \\
\text { es darum geht, die Hürde der Zertifi- } \\
\text { zierung zu überwinden. } \\
\text { Viele Prüfungen für detaillierte } \\
\text { Modulstruktur. }\end{array}$ & $\begin{array}{l}\text { Die Lernerfahrung steht im } \\
\text { Mittelpunkt, die sich aus eigenen } \\
\text { Interessen und selbstentwickelten } \\
\text { Fragestellungen speist. } \\
\text { Prüfungen finden in größerem } \\
\text { Rahmen zu übergreifenden Themen } \\
\text { und Kompetenzen statt. } \\
\text { Übergreifende Kompetenzen aus } \\
\text { größeren Zusammenhängen stehen } \\
\text { im Vordergrund. }\end{array}$ \\
\hline Prüfungen & $\begin{array}{l}\text { Viele Prüfungen sind an Modulen } \\
\text { orientiert und oft eher auf Reproduk- } \\
\text { tion von Wissen hin ausgelegt. }\end{array}$ & $\begin{array}{l}\text { Prüfungen sind kompetenzorien- } \\
\text { tiert, finden in größeren Abständen } \\
\text { und Einheiten statt, decken größere } \\
\text { Gebiete ab. }\end{array}$ \\
\hline $\begin{array}{l}\text { Organisations- } \\
\text { rahmen }\end{array}$ & $\begin{array}{l}\text { Institutionelle Struktur: Eine } \\
\text { Hochschule fungiert als Studienort/ } \\
\text {-anbieter }\end{array}$ & $\begin{array}{l}\text { Institutionelle Vielfalt: Mehrere } \\
\text { akademische Institutionen sind } \\
\text { beteiligt. } \\
\text { Studierende organisieren Studi- } \\
\text { enrahmen und den flexiblen, an } \\
\text { Bedürfnisse angepassten Studien- } \\
\text { prozess }\end{array}$ \\
\hline Reputation & $\begin{array}{l}\text { Die Reputation der Hochschule } \\
\text { bestimmt den Wert des Abschlusses } \\
\text { auf dem Arbeitsmarkt. }\end{array}$ & $\begin{array}{l}\text { Studierende dokumentieren ihre } \\
\text { Fähigkeiten und Erfahrungen eher } \\
\text { in Assessments, auch durch quali- } \\
\text { tative Elemente, wie beispielsweise } \\
\text { Portfolios. } \\
\text { Der Wert des Hochschulabschlus- } \\
\text { ses orientiert sich vor allem am } \\
\text { Praxisbezug des Studiums, den } \\
\text { dort gemachten und dokumentier- } \\
\text { ten Erfahrungen und demonstrier- } \\
\text { ter Handlungskompetenz. }\end{array}$ \\
\hline
\end{tabular}




\begin{tabular}{|c|c|c|}
\hline Dimension & $\begin{array}{l}\text { derzeitiges } \\
\text { Hochschulmodell }\end{array}$ & $\begin{array}{l}\text { zukünftiges } \\
\text { Hochschulmodell (postmodern) }\end{array}$ \\
\hline Durchlässigkeit & $\begin{array}{l}\text { Zwischen Schule, Berufsausbildung } \\
\text { und Hochschule existieren klare } \\
\text { Schwellen zwischen akademischen } \\
\text { und nichtakademischen Program- } \\
\text { men. } \\
\text { Die Durchlässigkeit ist nicht durch- } \\
\text { gängig gegeben. }\end{array}$ & $\begin{array}{l}\text { Durchlässiges Kontinuum } \\
\text { zwischen den Bildungsbereichen } \\
\text { Schule, Berufsausbildung und } \\
\text { Hochschule sowie den jeweiligen } \\
\text { anschlussfähigen Bildungsniveaus } \\
\text { der nationalen und Europäischen } \\
\text { Qualifikationsrahmen }\end{array}$ \\
\hline
\end{tabular}

\section{C2.2 Lernen neu denken: Leitkonzepte für das Lernen an der Hochschule der Zukunft}

Sowohl pädagogisch-didaktisch und auf die Lernmodelle der Hochschulbildung bezogen als auch organisatorisch werden sich Hochschulen weiterentwickeln. In diesem Kapitel werden wir zunächst beschreiben, welche pädagogisch-didaktischen Ansatzpunkte sich bieten.

\section{C2.2.1 Digital, vernetzt und informell}

Das Studium der Zukunft wird digitaler sein: Vernetzt, digital und informell. Es wird weitgehend von den Möglichkeiten digitaler Lernwelten Gebrauch machen und neben formellen Lernangeboten auch die volle Bandbreite informeller Lernmöglichkeiten in Anspruch nehmen - institutionenübergreifend und vollständig vernetzt. Digitale Lernwelten bestehen aus einem ganzen Bündel an Entwicklungen, Trends und Sichtweisen, die einen Wandel vom Lehren zum Lernen befördern. Eine neue Sichtweise auf vernetzte und offene Lernwelten verbindet im Wesentlichen fünf Charakteristika:

1. Dass Lernen immer, überall und in vielen unterschiedlichen Kontexten stattfindet, nicht nur im Klassenraum;

2. dass Lernenden die Rolle der Organisierenden zufällt;

3. dass Lernen ein Leben lang stattfindet, multiepisodisch und nicht (nur) an Bildungsinstitutionen gebunden ist;

4. dass Lernen in Lerngemeinschaften (sog. Communities of Practice (Wenger 1998)) stattfindet: Lernende treten Communities bei, sowohl formellen als auch informellen; 
5. dass Lernen vielfach informell und non-formal stattfindet, zu Hause, am Arbeitsplatz und in der Freizeit und nicht mehr lehrenden- und institutionenzentriert ist.

In diesem Verständnis bedeutet digital unterstütztes Lernen nicht mehr eine digitale Lernplattform zu nutzen, sondern unter Zuhilfenahme der verfügbaren Social Software eine neue Art der Lernplattform zu schaffen: Nicht mehr ein Learning Management System (LMS) als Materialinsel im Ozean Internet zu benutzen, sondern ein Tor zum Web zu schaffen. Der E-Tutor (Lehrende) greift nur noch als Wegweiser ein, indem sie/er kleine Lerninhalte (Microcontent) in einem Portal zur Verfügung stellt, die die Tür zum selbstgesteuerten Lernen aufstoßen, um die gestellten Lernziele zu erreichen. Diese werden mit den Lernenden ausgehandelt und zu Beginn z. B. via Blog-Eintrag oder Podcast festgehalten. Damit setzt sich die Lernumgebung nicht mehr aus einer einzelnen Anwendung, sondern aus mehreren individuell zusammengestellten und miteinander operierenden Tools zusammen. In diesem Zusammenhang wurde der Begriff des Personal Learning Environment (PLE) geprägt. In einem PLE findet die individuelle Reflexion der/ des Lernenden in Weblogs oder Podcasts sowie kollaborativem Arbeiten in Wikis statt. Somit ist Lernen nicht mehr nur der Transfer und Konsum von Inhalt und Wissen, sondern auch dessen eigenständige Produktion.

Langfristig gesehen kann sich so eine persönliche Lernlandschaft entwickeln, die ein ,interaktives Portal mit allen Zugängen zur persönlichen digitalen Welt“ (Kerres 2006) des Einzelnen darstellt. In einem steten Wissensproduktionsprozess aggregieren Lernende als Kuratorinnen und Kuratoren ihre Inhalte nach persönlichem Interesse, reflektieren diese und stellen sie individuell neu zusammen, teilen sie im gewünschten sozialen Kontext mit anderen.

Kerres weist schon 2006 darauf hin, dass bestehende E-Learning (1.0) Ansätze oftmals den Nachteil haben, dass Lernprogramme, aber auch moderne Lernplattformen von den Lehrenden mühsam mit Inhalten, viel Zeit und Geld befüllt werden müssen und dann oft zum Datengrab verkommen, während das echte Leben „sich heute nebenan, im Internet" abspiele (Kerres 2006). Mit den Werkzeugen des Web 2.0 lässt sich der Inhalt des Internets zur Lehre nützen, dessen Inhalt sich laufend von selbst generiert und regeneriert (ibid: 5). In diesem Modell tritt an die Stelle des Bearbeitens fertiger Kursmaterialien ein aktives und kreatives „rip, mix and learn“ (Richardson 2005). An die Stelle eines LMS könnten E-Portfolios treten, mit deren Hilfe Lernende ihre Lern- und Arbeitsprozesse selbst managen, dokumentieren und mit anderen austauschen. 


\section{Informelles Lernen}

Die Metapher des lebenslangen Lernens macht deutlich, dass Lernende nicht Dauerbesucherinnen und -besucher von Lehrveranstaltungen werden können, sondern, dass vielmehr neue Lernformen gefragt sind, die selbstgesteuert, schnell, flexibel und problemorientiert aufgebaut sind. Informelles Lernen, „das sich in mittelbaren Lebens- und Erfahrungszusammenhängen außerhalb des formalen Bildungswesens entwickelt" (Dohmen 2001), rückt wieder in den Mittelpunkt der Diskussion. Es umfasst - so viel ist heute bekannt - 70 bis 80 Prozent aller Lernaktivitäten. Jay Cross spricht in seinem letzten Buch „Informal Learning“ (2003) davon, dass nur 10 bis 20 Prozent in formalen Lernszenarien gelernt werden, dagegen 80 Prozent durch informelles Lernen. Er fordert eine Formalisierung informellen Lernens und eine Informalisierung formalen Lernens. Trotzdem wird der formalen Bildung heute noch eine weit größere Bedeutung zugeschrieben als der informellen (Cross 2003).

\section{Vernetztes Lernen}

Im Studium der Zukunft geht es darum, dass Lernende in sozialen Netzwerken - digital und analog - selbstbestimmt lernen. Aus (konstruktivistisch) lerntheoretischer Perspektive stellen die Fürsprecher dieser Art des Lernens eine Möglichkeit der Belehrung menschlichen Lernens grundsätzlich in Frage. Dies wird damit begründet, dass ein selbst gesteuertes System (Lernende) von seiner Umwelt nicht determiniert, sondern allenfalls gestört (perturbiert) und angeregt werden kann. Zudem wird argumentiert, dass Lernen nicht allein dadurch funktioniert, dass externe Anforderungen gestellt werden - Lernen, so die Vorstellung - kann nicht ohne den Lernenden geplant werden (vgl. Holzkamp 1993: 184). Das Konzept des selbstgesteuerten Lernens bekommt dabei eine enorme Bedeutung. Selbst gesteuertes Lernen wird oftmals als Oberbegriff für alle Lernformen verstanden, in denen die Lernenden ihren Lernprozess bzw. Aufgaben, Methoden und Zeitaufwand selber bestimmen (und/ oder mitentscheiden) und verantworten können (Deitering 1996: 45). Friedrich und Mandl (1997) verdeutlichen den Unterschied zwischen Selbstbestimmung und Selbststeuerung folgendermaßen:

\footnotetext{
„Selbstbestimmtes Lernen gibt den Lernenden die Möglichkeit, die Auswahlarten (was wird gelernt?) und die Lernziele (woraufhin?) eigenständig mitbestimmen zu können. Selbstgesteuertes Lernen enthält die Option von Lernenden den Weg des Lernens, die Lernregulation, (wie? wann?) bei vorgegebenen Lerninhalten und -zielen." (Friedrich \& Mandl 1997: 219)
}

Die grundsätzliche mediendidaktische Herausforderung ist es dabei, das didaktische Lernarrangement an den Parametern des didaktischen Feldes auszurichten, wie z. B. 
an den Merkmalen der Zielgruppe, der Spezifikation von Lehrinhalten und -zielen, didaktischen Methoden, didaktischer Transformation und Strukturierung der Lernangebote, Merkmalen der Lernsituation und Spezifikation der Lernorganisation, Merkmalen und Funktionen der gewählten Medien und Hilfsmittel (Kerres 2001). Es ist wichtig, auf das Primat der Didaktik hinzuweisen und zuerst die Frage nach den Bildungszielen zu stellen und erst dann geeignete Lehr/ Lernszenarien und -methoden zu wählen sowie die notwendigen Werkzeuge, um diese umzusetzen.

George Siemens entwickelte eine neue Lerntheorie, die 2004 veröffentlicht wurde: Konnektivismus. Er gibt an, sein Entwurf des Konnektivismus weise in seinen Prinzipien über die bisherigen lerntheoretischen Ansätze des Behaviorismus, Kognitivismus und des Konstruktivismus hinaus und berücksichtige dabei die zunehmende Tendenz des Lernenden hin zu informellem, vernetztem und elektronisch gestütztem Lernen. Das Lernen wird dabei gesehen als zunehmend kontinuierlicher, lebenslanger Prozess, der in alltägliche Arbeits- und sogar Freizeitaktivitäten eindringt und sowohl den Einzelnen als auch die Organisation und deren Verbindungen untereinander beeinflusst. Siemens führt aus, das Wissen über Wo? und Wer? sei heute wichtiger, als das Wie? und Warum? Obwohl Siemens Entwurf sich nicht klar gegen bestehende Lerntheorien abhebt, sondern eher eine netzwerkorientierte Lernphilosophie beschreibt, ist der Ansatz vor allem dahingehend wertvoll, dass er mit großer Klarheit die Entwicklung von vernetztem, digitalen Lernen und sozialen Prozessen als Grundlage für die stattfindenden Lern- und Interaktionsprozesse hervorhebt.

\section{2.2.2 Jenseits der Disziplinen}

Wie können gesellschaftliche Problemlagen zum Dreh- und Angelpunkt für Lernanlässe im Studium werden, damit Studierende unterschiedliche und teilweise konkurrierenden Lösungsansätze verschiedener Wissenschaftsdisziplinen kennenlernen und auf ihren Beitrag zur Lösung des Problems prüfen können? Probleme folgen keiner Disziplin - Studiengänge schon. Und damit ist ein grundlegendes Problem akademischer Differenzierung beschrieben. Zwar ist es sinnvoll und sogar unerlässlich, dass die unterschiedlichen Wissenschaftsdisziplinen einen eigenen Kern, eine eigene Identität, eigene Methoden, Inhalte, Forschungsrichtungen, Wissensbestände und Lehrtraditionen entwickeln und pflegen. Die Geschichte der Academia ist jedoch eine Geschichte der Differenzierung die oftmals eigene Blickwinkel mehr betont als Gemeinsame und die Frage nach dem Beitrag anderer Ansätze, Disziplinen und Methoden zur Lösung eines gesellschaftlichen Problems ausblendet. Im Ergebnis werden dadurch Hochschulbildungsprozesse gefördert, die 
stark auf eine Disziplin und ein Lösungsparadigma fokussiert sind, und die eine breite Orientierung und Fähigkeit zur Navigation in verschiedenen Wissenschaftsgebäuden nicht haben. Diese ist in der Regel allerdings notwendig, um Probleme - zumal gesellschaftliche - zu lösen. Sie ist notwendig, um die Frage zu beantworten, was die jeweils einzelne Wissenschaftsdisziplin zur Lösung eines spezifischen Problems eigentlich beiträgt, wie dieser Beitrag in Bezug auf alternative Beiträge anderer Wissenschaften und/ oder Disziplinen bewertet und gewichtet werden kann, und wo sich Lücken auftun, die Fragen an weitere Wissenschaften stellen. Die Methode der Problemorientierung ist der eigentliche Schlüssel im Hochschulstudium, um trans- und interdisziplinär wissenschaftliche Ansätze aufeinander zu beziehen (siehe Abbildung 36). Denn: Probleme folgen keiner Disziplin.

Inter- und transdisziplinäres Lehren und Lernen erfordert:

- Verknüpfung und Anwendung des Erlernten in konkreten und realen Aufgabenstellungen,

- eine aktive Auseinandersetzung mit praktischen Fragestellungen,

- Erarbeitung von interdisziplinären Lösungsansätzen,

- gemeinsames Bearbeiten von Themen aus Gesellschaft und Wirtschaft.

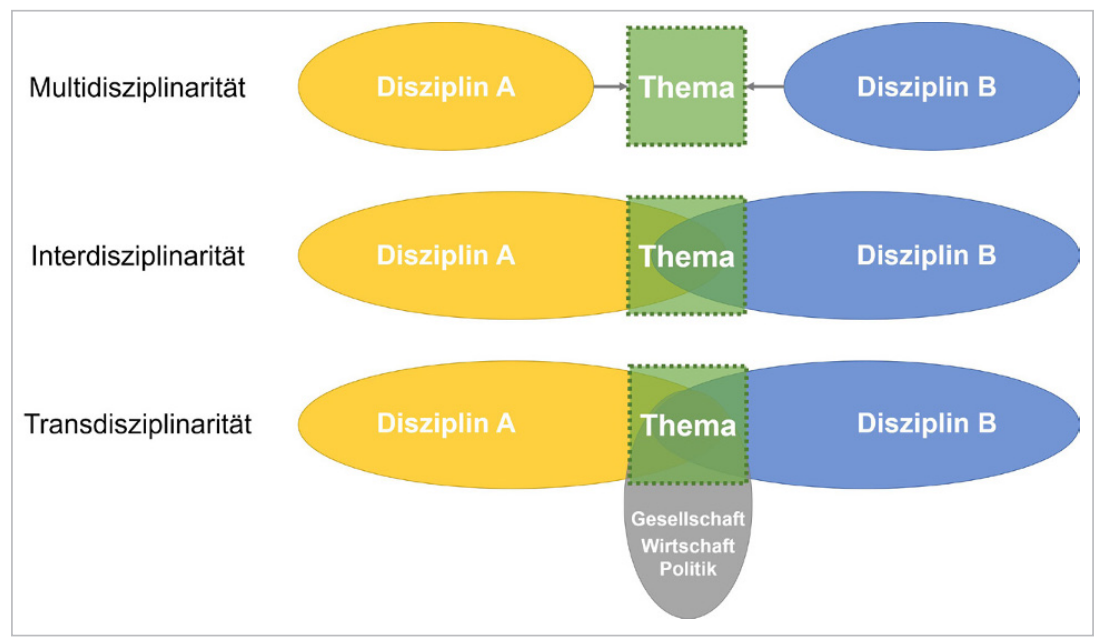

Abb. 36 Inter- und transdisziplinäres Lernen 
Es ist daher unerlässlich, dass Absolventinnen und Absolventen eine interdisziplinäre und oder transdisziplinäre Grundhaltung und die Kompetenz zur kreativen Analyse von Problemstellungen auf Basis von methodologischem Handwerkszeug verschiedener Wissenschaftsrichtungen erlernen können. Dabei müssen sie keine Fachexpertinnen und Fachexperten der jeweiligen Wissenschaftsdomäne sein, jedoch geübt im Blick auf die unterschiedlichen Beiträge sein, die verschiedene Wissenschaften zu einem jeweils definierten Problem beitragen können.

\section{Gute Praxisbeispiele}

- HOTSPOT (House of Transdisciplinary Studies for practice-oriented teaching and learning) an der Hochschule Pforzheim.

- Fächerübergreifende Bachelorstudiengänge an der Universität Hannover

\section{C2.2.3 Individuelle Studienpfade}

Wie können Curricula mit Inhalten von Studiengängen anderer Hochschulen angereichert werden, die digital zur Verfügung gestellt werden, also gewissermaßen digital importiert werden? Eine weitere Möglichkeit, Studienverläufe interdisziplinärer und flexibler zu gestalten, mehr Wahlmöglichkeiten zuzulassen und die Selbstorganisation der Studierenden zu stärken, ist der digitale Import von Curricula anderer akademischer Institutionen, auf Englisch mittlerweile auch virtual Erasmus oder virtual mobility bezeichnet. Studierende belegen in diesem Fall eine Lehrveranstaltung, eine Sommerschule oder absolvieren ein Praktikum, welches in Form eines Onlinekurses an einer anderen akademischen Einrichtung (oft auch im Ausland) als der Hochschule, bei der sie eingeschrieben sind, vermittelt wird. Das so belegte Studienangebot wird als vollwertige Studienleistung anerkannt und kann in das eigene Studium mit allen Leistungspunkten integriert werden. Der digitale Import von Lehre aus anderen akademischen Einrichtungen kann begünstigt werden, wenn Fakultäten sich von vornherein um die möglichen Importmöglichkeiten Gedanken machen, Regeln dazu aufstellen und diese in einem Wahlkatalog für virtuelle Mobilität für die Studierenden beigefügt werden. ${ }^{40}$

Eine Variante der oben beschriebenen Flexibilisierung des Studienverlaufs ist auch eine stärkere interdisziplinäre Gestaltung von Studienangeboten durch den Einsatz digitaler Medien. Dabei werden flexible Wahlbereiche definiert, um Module und

40 Das EU Projekt „OER Test“ hat die hierbei denkbaren Möglichkeiten aufgearbeitet und publiziert: https://oerknowledgecloud.org/sites/oerknowledgecloud.org/files/Open-Learning-Recognition.pdf 
Veranstaltungen aus anderen und auch fachfremden Fachbereichen zu studieren. Denkbar sind Beispiele wie die Theologin, die auch Managementseminare belegen möchte, der Manager, der an Gruppenpsychologie interessiert ist, etc. Hochschulen beginnen Module aus Bachelor- und Masterstudiengängen als sogenannte , polyvalente Module zu definieren. Das hat Auswirkungen auf die Kapazitätsberechnung und Auslastung von Studiengängen. Digitalisierung ermöglicht eine zeit- und ortsunabhängige Präsentation und Erreichbarkeit von entsprechenden Inhalten, auch über Fakultäts-, Department-, Campus- und sogar Hochschulgrenzen hinweg. Ein Beispiel hierfür ist etwa die Virtuelle Hochschule Bayern, über die viele bayrische Universitäten mittlerweile über 300 Lehrveranstaltungen und Module digital anbieten.

\section{2.2.4 Soft Skills als harte Währung}

Es kann nicht oft genug betont werden, dass Future Skills auch die Vermittlung von relevantem Wissen miteinbeziehen - aber eben mit Wertvorstellungen, Haltungen und Handlungsdispositionen imprägniert. In der Agenda für die Hochschullehre der Zukunft lösen Future Skills also nicht die Vermittlung von Wissen ab, transponieren Wissen jedoch - ganz im Sinne des in Abbildung 32 vorgestellten Stufenmodells - auf eine höhere Stufe. Future Skills werden in der zukünftigen Hochschullehre gleichwertig neben Wissenstransferkonzepte treten (siehe Abbildung 37)- so bewerten die Expertinnen und Experten des internationalen NextSkills Delphi diesen Aspekt $(M=4,16, S D=0,70, A=91,1 \%, N=45)^{41}$. Die Bedeutung, die sowohl die Interviewten der NextSkills Studie als auch das Expert(inn)enpanel in der NextSkills Delphi-Studie geben, lässt fraglich erscheinen, ob der Begriff Soft Skills tatsächlich noch tragfähig für die als Future Skills beschriebenen Kompetenzen ist. Wird doch mit der Einleitung in Soft und Hard oftmals auch vermittelbar und weniger vermittelbar suggeriert, bzw. gut zu prüfen und weniger gut zu prüfen verbunden. Tatsächlich scheint hierbei auch eine Hauptbarriere für die flächendeckende Umsetzung einer Orientierung von Hochschulcurricula an Future Skills zu liegen - darin nämlich, dass Prüfungssysteme bislang vor allem auf die Prüfung von Wissen ausgelegt sind und nicht auf die Prüfung von Handlungskompetenzen.

Während etwas mehr als vier von zehn Befragte angeben, dass Future Skills heute bereits gleichwertig neben die reine wissensorientierte Vermittlung treten, sehen fast die Hälfte der Befragten die Umsetzung von Future Skills als Leitorientierung in einem Fünfjahreszeitraum, bzw. jeder zehnte Befragte in einem Zehnjahreszeitraum.

41 A bezeichnet den Agreement-Index als Anteil derjenigen die stark zugestimmt (=5) bzw. zugestimmt haben $(=4)$. 


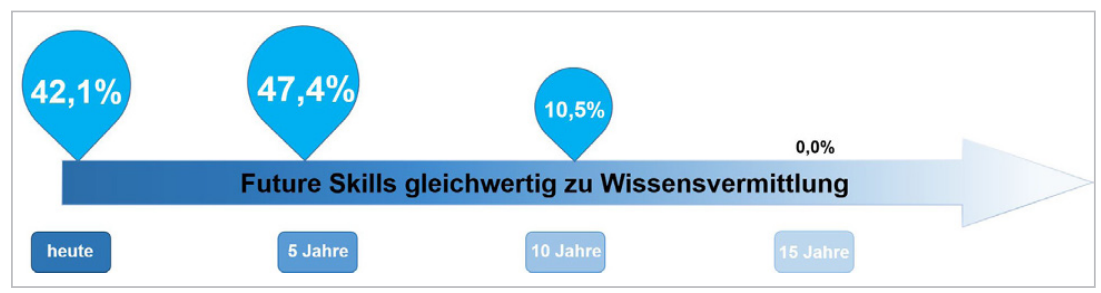

Abb. 37 Geschätzte Zeitdauer für die zunehmende Wichtigkeit von Future Skills als gleichwertig zu Wissensvermittlung $(\mathrm{N}=38)$

Als neues Leitziel für eine zukünftige Hochschullehre geben die Befragten der NextSkills Studie die Handlungsfähigkeit in hochemergenten Handlungskontexten an - also Future Skills. Dabei steht der Umgang mit Situationen unvollständiger Informationslage und in potentiell unsicheren Situationen im Vordergrund. Die Fähigkeit sich in unbekannten und komplexen Zukunftskontexten zurechtzufinden, wird zur Hauptorientierung in der Hochschulbildung und damit wichtiger als Wissensvermittlung. Diese Einschätzung bekommt bei Expertinnen und Experten eine hohe Zustimmung: $M=3,64, S D=0,99, A=62,2 \%, N=45$. In der Abfrage der time of adoption geben fast fünf von zehn befragten Expertinnen und Experten des NextSkills Delphi an, dass Handlungsfähigkeit in emergenten Kontexten bereits heute ein wichtiges, bestimmendes Feld für die Gestaltung von Hochschullehre sei. Diese Entwicklung wird sich innerhalb der nächsten zehn Jahre weiter verstärken (siehe Abbildung 38).

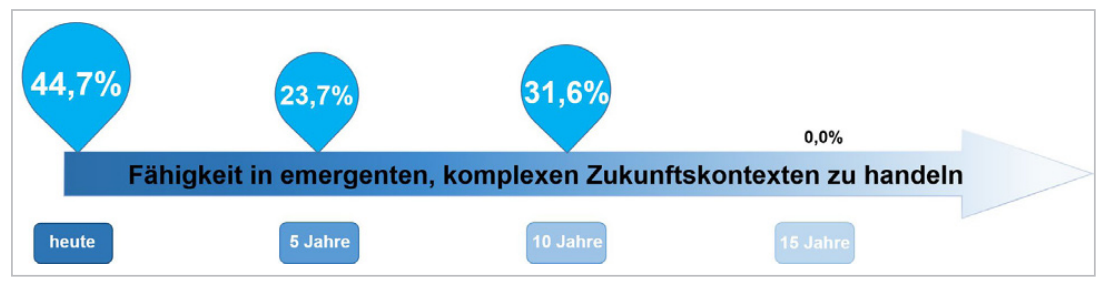

Abb. 38 Geschätzte Zeitdauer für die zunehmende Wichtigkeit der Fähigkeit, in emergenten, komplexen Zukunftskontexten handeln zu können $(\mathrm{N}=38)$ 


\section{2.2.5 Vom defensiven zum expansiven Lernen}

Wie können Hochschulen die Fiktion aufgegeben, dass Lernprozesse vollständig durch Planung, Curricula und didaktische Lehrprozesse vorausgeplant werden können? Wie kann die Vision einer studierendenzentrierten Lehre im Sinne des shifts from teaching to learning wirklich vollzogen werden? Das Verständnis, Lernens als aktiven und intentionalen Prozess zu verstehen, wurde im Rahmen der kritischen Lerntheorie des Hamburger Professors Klaus Holzkamp entwickelt und formuliert. Dabei steht der Begriff des expansiven Lernens für Lernen, welches aus eigener Intention und eigenem Interesse geschieht und der Überwindung subjektiv empfundener Handlungsbarrieren dient. Holzkamp (1995) formuliert, dass intentionales Lernen sich wiederrum in expansives und defensives Lernen unterscheidet. Expansives Lernen meint dabei die Art von Lernen, die auf eine Verbesserung der eigenen Lebensqualität abzielt, zur Überwindung subjektiv empfundener Handlungsbarrieren - nicht zu verwechseln mit der intrinsischen Motivation nach dem Motto wolle, was du sollst. Vielmehr geht es um den lernenden Weltaufschluss im Gegensatz zur defensivem Lernbemühung. Diese meint Lernen als Abwehr von bevorstehender Bedrohung und dient damit der Problemvermeidung (vgl. Holzkamp 1995: 190ff.).

„Holzkamp hat die Vorstellung, man könne durch Lehrpläne, Lehrstrategien oder didaktische Zurüstung die Lernprozesse eindeutig vorausplanen, als Fiktion kritisiert. Eine Didaktik jenseits des Lehr- Lern-Kurzschlusses muss also alle Herstellungsillusionen (...) aufgeben." (Faulstich 2008: 56)

Für die individuelle Kompetenzentwicklung müssen Lernsituationen geschaffen werden, in denen selbstgesteuertes, anwendungsbezogenes, situatives, emotionales, soziales und kommunikatives Lernen gefördert werden (Mandl \& Krause 2001). Die Integration komplexer und authentischer Probleme in unscharfen Ausgangssituationen ist dabei ein wesentliches Element in kompetenzorientierten Lernszenarien. Lerndesigns werden sich zukünftig zunehmend von Präsentationsund Wissenstransfer-Methoden abwenden und sich stattdessen auf interaktive sozio-konstruktive Ansätze fokussieren $(M=3,76, S D=0,76, A=64,5 \%, N=45)$ (siehe Abbildung 39). 


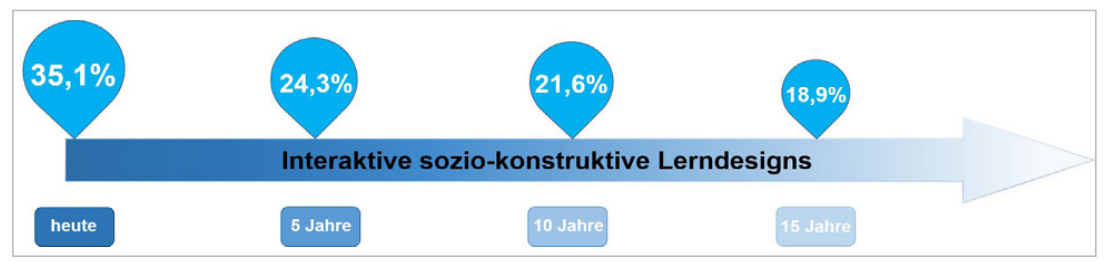

Abb. 39 Geschätzte Zeitdauer für die zunehmende Wichtigkeit interaktiver soziokonstruktiver Lerndesigns $(\mathrm{N}=37)$

Dabei können gerade auch digitale Medien unterstützend eingesetzt werden: Digitale Lernumgebungen können Studierende unterstützen, jenseits der Beschäftigung mit künstlich aufgearbeiteten Fragestellungen im Seminarraum, digital mit Betroffenen, Akteurinnen, Akteuren, Expertinnen und Experten in Kontakt zu kommen und sich zusätzlich zum theoretischen Wissensbestand ein reales, authentisches Problemszenario zu erarbeiten. In der Hamburg Open Online University (https://www.hoou.de) wird diese Verzahnung von akademischer Analyse und realer Problemwelt anhand von vielen Projekten real angegangen, indem Studierende über Lernplattformen mit Protagonistinnen und Protagonisten der jeweiligen Themenfelder zusammenarbeiten. Weiterhin können digitale Medien auch hier Möglichkeiten bieten, individuelle Reflexionen per Videotake oder über reflexives Schreiben, beispielsweise in Weblogs, zu praktizieren und in die Hochschullehre zu integrieren.

Zusätzlich werden kollaborative Lernszenarien in denen Lernende miteinander lernen an Bedeutung gewinnen, anstelle einer Orientierung auf Wissenstransfer (Vorlesungsformate). Diese Prognose geht aus dem NextSkills Delphi mit hohen Zustimmungswerten hervor $M=3,71$ und $A=60,0 \%(S D=0,91, N=45)$. Während viele Expertinnen und Experten diese Lernszenarien bereits heute als bedeutsam einschätzen (39,5\%), sieht jeder Dritte (34,2\%) diese Entwicklung als erst in zehn Jahren als realistisch an (siehe Abbildung 40).

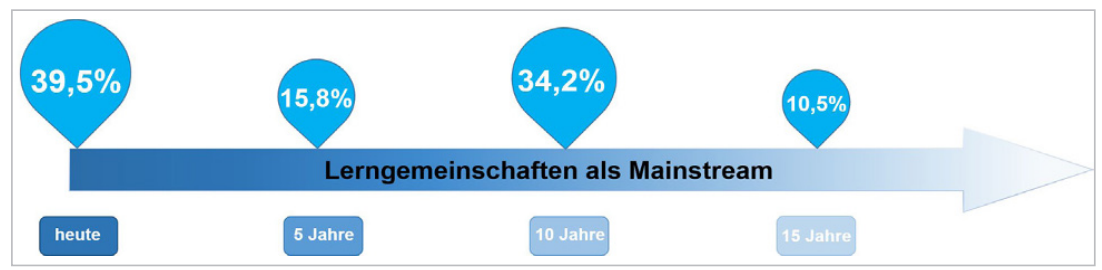

Abb. 40 Geschätzte Zeitdauer für die zunehmende Wichtigkeit von Lerngemeinschaften als Mainstream $(\mathrm{N}=38)$ 


\section{C2.2.6 Zukunft des Prüfungswesens}

Wie kann die oft auf Reproduktion von Wissen hin orientierte Prüfungspraxis zugunsten von kompetenzorientierten Prüfungsformen und von Peer-Validierungsmodellen weiterentwickelt werden? Im Sinne des Ansatzes des Constructive Alignments (Biggs \& Tangs 2011) sind kompetenzorientierte Lehr- und Lernszenarien nur dann sinnvoll, wenn auch die Prüfung und Bewertung kompetenzorientiert vorgenommen wird. Es ist klar, dass diese Prüfungsformen zukünftig an Relevanz gewinnen. Dabei treten Bewertungen von Lernen um des Lernens Willen (formative und Peer-Bewertung) an die Stelle der Bewertung des Gelernten (summative Bewertung) (siehe Abbildung 41, $M=3,80, S D=0,86, A=66,7 \%, N=45$ )

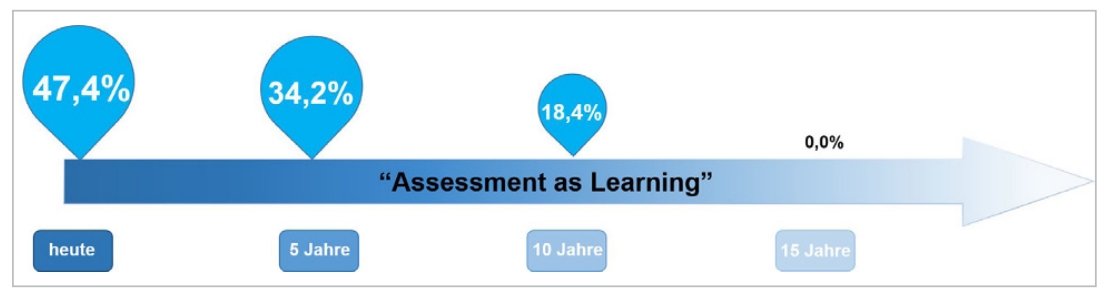

Abb. 41 Geschätzte Zeitdauer für die zunehmende Wichtigkeit eines Assessments as Learning $(\mathrm{N}=38)$

In der Hochschuldidaktik wird dieses Thema bereits stark fokussiert, in der Realität der Hochschullehre ist es oftmals zugunsten von Massenprüfungen im Sinne eines „Auswendiglernen und Wiedergeben“, einem eher reproduktiven Verständnis folgend, noch nicht sehr verbreitet. Dies ist auch für Hochschulbildung digital eine Herausforderung. Aber insgesamt gilt: Nur wenn auch die Prüfungsformen und -inhalte an Kompetenzen und Future Skills orientiert sind und nicht auf Wissensabfragen reduziert werden, ist es sinnvoll, Future Skills auch als Leitkonzept für die hochschulischen Lernprozesse in den Blick zu nehmen. Digitale Medien werden seit längeren auch für Prüfungs- und Bewertungsprozesse herangezogen. Die in 2015 erschienene Studie „Digitales Prüfen und Bewerten“ (Michel et al. 2015) gibt einen strukturierten Überblick über den Stand der Dinge und die Vielfalt der (teil) digitalisierten Prüfungsformate, die derzeit bereits an Hochschulen zum Einsatz kommen.

Eine kritische Anmerkung sei mit Gabi Reinmann (2014) an dieser Stelle gestattet: Die Ausrichtung von Hochschulbildung an Future Skills wirklich zu Ende 
zu denken, würde bedeuten, nur Prüfungen zuzulassen, die tatsächlich versuchen, Future Skills und Kompetenzen zu erfassen, und die Anforderungen integrierter Prüfungen erfüllen. Dabei ist allerdings Bescheidenheit geboten, denn bislang bleiben kompetenzorientierte Prüfungen ein Ideal, dem man sich nur annähern kann. Präzise und eindeutige Feststellungen, über welche Kompetenz bzw. über welche Future Skills - im Sinne einer Handlungsdisposition - jemand in welcher Ausprägung verfügt, sind theoretisch und praktisch kaum möglich. Denn Kompetenzen stellen eben Handlungsdispositionen dar und nicht vollständig in sich abgeschlossene, vorbereitete und abrufbare fertige Handlungsabläufe. Notwendig sind komplexe Prüfungen, die Kompetenzen mit einem reflektierten Anspruch erfassen. Sowohl die Gestaltung als auch die Durchführung solcher Prüfungen sind sehr aufwändig. Es zeigt sich, dass kompetenzorientiertes Prüfen ein aufwändiges Vorhaben ist. Bei diesem können jedoch gerade auch digitale Medien fruchtbar eingesetzt werden. Im Sinne des Ideals der Universitas würden dann auch nicht mehr ein Abprüfen von Auswendiggelerntem im Vordergrund stehen, sondern die Disputation von Neuem und Bemerkenswerten.

Ziel ist es, vom oftmals vorherrschenden Frage-Antwort-Spiel wegzukommen, hin zu einem Austausch zwischen Studierenden und Lehrenden auf Augenhöhe. Um dies zu ermöglichen, können die Prüflinge bei der Auswahl der Themen miteinbezogen werden, beispielsweise über ein Portfolio. Ein Portfolio ist eine Sammlung von Dokumenten, die der Studierende im Verlauf der Lehrveranstaltung(en) eigenständig erarbeitet. Es stellt somit die Auseinandersetzung und den aktiven Umgang mit wichtigen Seminarinhalten dar. Ein solches Portfolio ist eine geeignete Grundlage für ein Prüfungsgespräch über die im Portfolio dargestellten Inhalte (Wildt, J. \& Wildt, B. 2011). Studierende werden somit deutlich stärker in die Gestaltung der Prüfung mit einbezogen, indem sie das Portfolio selbst zusammenstellen und damit Einfluss auf die relevanten Prüfungsinhalte ausüben und darüber hinaus als Produzent der Portfoliotexte auch in einer Rolle als Experte/ Expertin für deren Inhalte angesehen werden können.

In der Realität der Hochschulen ist es heute so, dass im Rahmen eines Bachelorstudiums etwa 20 bis 30 Module studiert werden. Jedes Modul schließt mit einer Prüfung ab. Dabei werden in der Regel mehrere Fächer im Rahmen der Prüfung geprüft, was faktisch einer Unterteilung der Prüfung in mehrere Teilprüfungen gleicht (Pietzonka 2014). Dadurch haben Studierende heute im Rahmen ihres Bachelorstudiums faktisch ca. 50 bis 60 Prüfungsteile zu leisten - davon geht jedes in die Abschlussnote ein (vgl. z. B. Wannemacher \& Kleimann 2010). Gabi Reinmann (2014) beschreibt dies so: Die psychische Ausnahmesituation für Studierende in der letzten Phase des Studiums der alten Studiengänge (noch vor der Bolognareform) erstreckt sich heute auf die gesamte Dauer des Studiums (Bülow-Schramm 
2008: 31). Man hat sich also von einem Extrem ins andere bewegt: Den einen alles entscheidenden Prüfungszeitpunkt am Ende des Studiums hat man gegen einen alles beherrschenden Prüfungszeitraum vom Anfang bis zum Ende des Studiums eingetauscht. Reinmann führt weiter an, dass Kompetenzorientierung zu Ende denken (vgl. auch Bülow-Schramm 2008: 39), heißen würde, nur Prüfungen zuzulassen, die tatsächlich versuchen, Kompetenzen zu erfassen. Zu paaren ist das aber mit Bescheidenheit, da man sich dem nur annähern kann. Huber (2008: 22) hält kompetenzorientierte Prüfungen gar für utopisch.

In jedem Fall werden kompetenzorientierte Prüfungen komplexere Verfahren sein, in denen in der Regel offenere schriftliche, mündliche und forschungspraktische Formate zum Einsatz kommen müssen. Da dies viel aufwändiger ist als die heutige Prüfungspraxis, könnte ein entsprechend ausgerichtetes Studium in dem kompetenzorientierte Prüfungsverfahren zum Einsatz kommen, nur noch viel weniger Prüfungen umfassen. Reinmann (2014) kommt zu folgendem Schluss:

„...die optimale Anzahl dieser ,Prüfungen mit Rechtsfolgen' [hängt] von der Fachrichtung [ab], [müsste] unabhängig davon aber im einstelligen Bereich bleiben (...). Davon ausgenommen sind formative Leistungsnachweise, die ausschließlich dazu dienen, Studierenden eine Rückmeldung auf ihren Lernprozess und bereits erworbene Kompetenzen zu geben. Sie haben keinen Einfluss auf die Abschlussnote und sind Teil didaktischer Szenarien."

Die Weiterentwicklung der Prüfungsformate stellt einen wichtigen Zukunftsbaustein der Lehre in der Hochschule der Zukunft dar - so schätzen die Befragten der NextSkills Delphi-Studie, dass Hochschulbildung innerhalb der nächsten fünf bis zehn Jahre verstärkt auf Lernen durch Peer-Validierung bauen wird $(M=3,73, S D$ $=0,90, A=62,2 \%, N=45$ ) (siehe Abbildung 42).

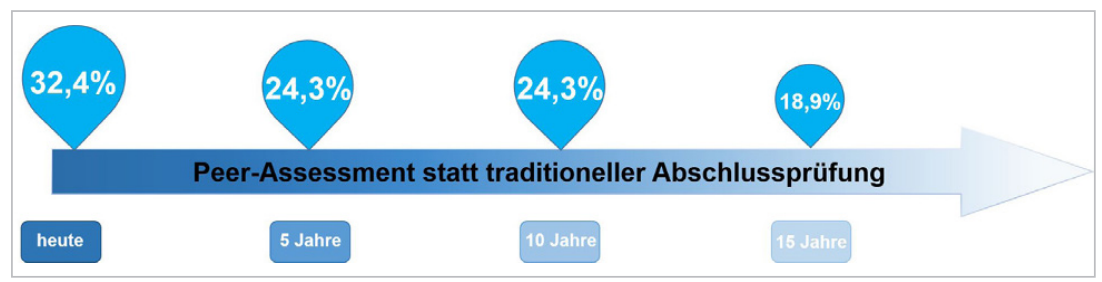

Abb. 42 Geschätzte Zeitdauer für die zunehmende Wichtigkeit von Peer-Assessment statt traditioneller Abschlussprüfungen $(\mathrm{N}=37)$ 


\section{C2.3 Die Hochschulorganisation neu denken: Eckpfeiler der Hochschule der Zukunft}

\section{2.3.1 Digital: Jenseits der Technisierung}

Wie können Hochschulen Strategien für die digitale Transformation entwickeln, die Digitalisierung nicht als Technisierung versteht, sondern als Aufruf, Lehre, Lernen und Studium neu zu denken und weiter zu entwickeln? Digitale Medien bieten der Hochschule neue Möglichkeiten, die Lehre personalisierter und unabhängig von Zeit und Ort zu gestalten. Die Ergebnisse der jüngsten Debatte über Hochschulbildung digital zeigen, dass Digitalisierung nicht als Technisierung, sondern als Ermöglicher für didaktische Phantasie in der Lehre steht (Hochschulforum Digitalisierung 2016). Zu erkennen ist, dass es Hochschulen darum geht, junge Menschen in der Entwicklung ihrer Fähigkeit zur selbstständigen und eigenverantwortlichen Arbeit in heterogenen Teams zu unterstützen und sie bei der Entwicklung von Handlungskompetenzen durch die Lösung komplexer Probleme zu fördern. Digitalisierung ist hier ein Ermöglicher, ein Impulsgeber für die Lehre der Zukunft. Hochschulen und ihre Akteure in der Lehre setzen digitalen Medien in großer Vielfalt ein und nutzen die sich dadurch ändernden Rahmenbedingungen, um produktiv neue Wege zu gehen. Dabei wird Hochschullehre jenseits von reinem monodirektionalem Wissenstransferkonzepten und Massenveranstaltungen attraktiv gestaltet. Dann wird Hochschullehre zum Reallabor, in dem Konzeptionen entwickelt und umgesetzt werden, in denen Studierende als reflektierende Praktikerinnen und Praktiker in „Reflexionslaboratorien“"(Ehlers 2014) lernen, in denen sie kollaborativ zusammenarbeiten und in denen sie in ihrer Entwicklung zu autonomen und selbstgesteuerten Lernenden unterstützt werden. Digitalisierung verfolgt dabei nicht das Ziel der Technisierung, sondern fordert zur didaktischen, curricularen und organisatorischen Innovation in der Lehre auf.

\section{2.3.2 Vom Studienfach zur Mission}

Wie können Hochschulen Curricula flexibilisieren und individualisieren und Möglichkeiten des Build your own Curriculum realisieren? Hochschulstudiengänge sind heute von großer innerer Geschlossenheit gekennzeichnet. Dabei wird versucht ein in sich geschlossenes System von aufeinander abgestimmten Qualifikationszielen innerhalb von sechs, acht (Bachelor) und/ oder vier Semestern (Master) zu integrieren. Zumeist liegt ein längerer Analyseprozess eines Berufsfelds zugrunde, aus dem die relevanten Qualifikationsziele abgeleitet werden. Ziel ist dabei das Erreichen eines 
klar definierten Gesamtabschlusses für das Studium. Dabei werden die Abschlussbezeichnungen hoheitlich von einer Hochschule vergeben. In Zeiten in denen konkrete Qualifikationsziele immer weniger aus Berufsfeldanalysen generiert werden können, da diese selber einer starken Veränderungsdynamik unterliegen, stellt sich die Frage, ob die derzeit vorherrschende akademische Grundeinheit Studiengang noch adäquat sein wird. Die internationale Delphi-Studie des NextSkills Projektes kommt hierbei zum Schluss, dass Studieren in der Zukunft anders aussehen wird. Es wird dabei von multi-institutionellen Studienverläufen ausgegangen, in denen Studierende zwischen unterschiedlichen Hochschulen hin- und herwechseln und Lehrveranstaltungen bei verschiedenen Hochschulen belegen. Auch binnenstrukturell wird von Veränderungen im Studienverlauf ausgegangen. Die Expertinnen und Experten gehen mit hohen Zustimmungswerten $(M=3,6, A=60,0 \%)$ davon aus, dass ein Hochschulstudium keinem klar vorgegebenen Studienplan mehr folgt, sondern sequentiell oder parallel an mehreren Hochschulen vonstattengeht $(S D=$ $0,84, N=45) .{ }^{42}$ Dadurch entsteht ein institutionelles Studienerfahrungs-Patchworkmuster. Mehrere akademische Institutionen sind beteiligt und die Studierenden organisieren den Studienrahmen flexibel und an ihre Bedürfnisse angepasst.

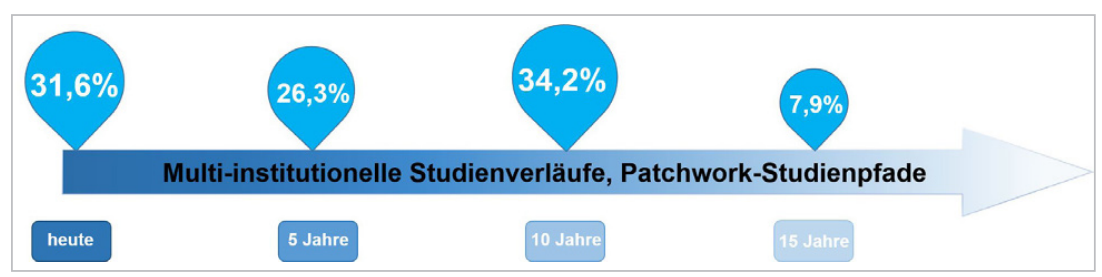

Abb. 43 Geschätzte Zeitdauer für die zunehmende Wichtigkeit multi-institutioneller Studienverläufe \& Patchwork-Studienpfade $(\mathrm{N}=38)$

Das Studium setzt sich dann aus kleinen Studieneinheiten zusammen, die auch von unterschiedlichen (Hochschul-)Anbietern kommen können. Es wird mehr Kurzformate, mehr Zertifikatskurse, Kontaktstudienmöglichkeiten und mehr Short-Courses geben. Daras entstehen Patchwork-Studienverläufe, die dann zu größeren Abschlusszertifikaten wie beispielsweise einem Studienabschluss, zusammengefügt und von einer Hochschule zertifiziert werden können. Über neun von

42 Die Expertinnen und Experten wurden gebeten, die folgende Aussage einzuschätzen: „Studierende werden sequentiell oder parallel an mehreren Hochschulen studieren, wodurch sich ihr Studium als institutionelles Studienerfahrungs-Patchwork darstellt.“ 
zehn Befragten gehen davon aus, dass sich innerhalb eines Zeitraumes von zehn Jahren hierbei starke Veränderungen ergeben (siehe Abbildung 43).

Immer mehr Hochschulen bieten bereits sogenannte Wahlcurriculum-Optionen an. ${ }^{43}$ Die Jacobs-Universität in Bremen bietet ein sog. „3-C Model“ an ( Choice Core - Career), bei dem Studierende mit hohen Graden an Wahlfreiheit ihr eigenes Curriculum zusammenstellen können. Ein Foundation-Jahr ermöglicht zunächst Orientierung über Interessen einerseits und die akademischen Disziplinen, Fragestellungen und Methoden erlangen zu können bevor Studierende dann ihren Studiengang wählen. In Hochschulen, die eine Möglichkeit für Studierende anbieten, ihr eigenes Curriculum zusammenzustellen erfordert dies zumeist, dass diese einen schriftlichen Vorschlag bei einem Curriculum-Komitee einreichen, welcher dann geprüft und beraten wird; so beispielsweise an der Universität Maryland oder Michigan in den USA.

Für Hochschulen ergeben sich bei diesen Build Your Own Curriculum (BYOC) Ansätzen neue Herausforderungen. Sie müssen entsprechende Beratungs-, Begleitungs- und Coachingangebote bereithalten. Diese müssen professionalisierte Services sein, die sowohl von Professorinnen und Professoren als auch von Lerncoaches angeboten werden, die Studierende bei zunehmend diversen Lernerfahrungen sowohl bei der Reflexion, als auch Anwendung und Integration der Lerninhalte in Bezug auf größere Sinnabschnitte, Problemstellungen und Future Skills, sowohl disziplinär als auch interdisziplinär begleiten und betreuen.

\section{C2.3.3 Mehr Anerkennung und Anrechnung}

Wie können Hochschulen mehr Expertise und Professionalität bei der Anrechnung und Anerkennung von Vorerfahrungen und -leistungen entwickeln, um flexibleres Studieren und eine höhere Durchlässigkeit zu ermöglichen? Hochschulen in Deutschland sind verpflichtet, Kompetenzen aus dem akademischen (bis 100 Prozent) und nicht-akademischen (bis 50 Prozent) Bereich als Vorkenntnisse im Studium auf die zu erbringenden Prüfungsanforderungen anzuerkennen. Die Erfahrung mit solchen Anerkennungspraktiken ist jedoch nicht groß und führt oftmals zu Unverständnis auf Seiten der Lehrenden, da unklar ist, ob die zur Anerkennung eingebrachten

43 Die Website collegechoice.net listet 20 private und öffentliche Universitäten aus den USA auf, die für Studierende mit besonders guten Eingangsnoten Bachelorstudiengänge anbieten, bei denen Studierende - mithilfe von „Academic Advisors“ und „Study-Coaches“ - ihr eigenes Curriculum zusammenstellen können (https://www.collegechoice. net/best-bachelors-programs-design-your-own-major/). 
Vorleistungen auch wirklich adäquate Kompetenzen beinhalten (Hanft et al. 2014). Anerkennung und Anrechnung ist aber der wesentliche Schlüssel für die Ermöglichung neuer (digitaler) Vielfalt für Studienverläufe. Digitale Lehrveranstaltungen (unterschiedlicher Fakultäten und akademischer Institutionen) können erst dann vollwertig neben in Präsenz erbrachte Lehrveranstaltungen treten, wenn sie auch vollwertig anerkannt werden. Es erfordert jedoch in vielen Fällen die Ausweitung der Anerkennungspraxis. Dabei sind sowohl individuelle (Anerkennung individuell eingebrachter Leistungen) als auch institutionalisierte Konzepte (Kooperationsmodelle, in denen die von anderen Einrichtungen anerkennungsfähigen Leistungen im Vorfeld geprüft wurden) denkbar (ebenda). Im internationalen Delphi des NextSkills Projekt sind sich die Expertinnen und Experten weitgehend einig: Über 75 Prozent gehen davon aus, dass innerhalb der nächsten fünf Jahre eine starke Zunahme an episodischen Patchworkverläufen der Studienerfahrungen zu beobachten sein wird, in denen bereits erbrachte Leistungen und vorhandene Kompetenzen anerkannt werden. $(M=3,59, S D=0,96, A=59,1 \%, N=44)$.

\section{2.3.4 Microcredentials: Alternative Zertifizierungsmethoden}

Wie können Hochschulen mit Microcredentials und alternativen Zertifizierungsverfahren Erfahrung gewinnen, um Studieren zunehmend anschlussfähiger, durchlässiger und flexibler zu gestalten? Während in heutigen Hochschulmodellen Lehre und Prüfung, sowie Prüfung und Zertifizierungsprozesse aneinandergekoppelt sind, werden diese Prozesse in Hochschulmodellen der Zukunft immer stärker entflochten und voneinander unabhängig sein. Diese Entkoppelungsprozesse von bislang weitegehend strukturell gekoppelten und zusammengehörenden Vorgängen der Vermittlung, Prüfung sowie der Zertifizierung birgt Chancen und Herausforderungen. Chancen liegen in der Flexibilisierung von Studienverläufen, ganz im Sinne des oben beschriebenen individuellen Patchworkstudienmusters. Führt akademisches Lernen nicht immer nur ausschließlich auf Prüfungen und Testungen hin, so steht auf einmal der reine Lernprozess im Mittelpunkt der Bemühungen. Lernen wird intrinsischer und kann sich auch jenseits und unabhängig von prüfbaren oder in Prüfungsordnungen verzeichneten Inhalten bewegen. Gleichzeitig wird eine Begleit- und Reflexionsfunktion wichtiger, die Lernen in akademischen Kontexten daraufhin orientiert, welchen Beitrag die/ der Lernende leistet, um die eigenen, zuvor wahrgenommenen Handlungsbarrieren zu überwinden. Digitale Lehre macht eine Flexibilisierung von Raum und Zeit möglich und begünstigt daher auch das Wahrnehmen von Studienerfahrungen gleichzeitig an verschiedenen Hochschulen sowie in verschiedenen Modi - sowohl physisch 
in Präsenz als auch online virtuell, sowohl offiziell eingeschrieben als auch als Teilnehmerin oder Teilnehmer an einem offenen Onlinekurs. In der Befragung des internationalen Delphis stimmen die Teilnehmerinnen und Teilnehmer der Aussage weitgehend zu, dass Studienerfahrungen, die in kleinen, modularisierten Einheiten (Microcredentials) zertifiziert werden, zukünftig die gleiche Bedeutung bekommen werden, wie die Zertifizierung gesamter Studiengänge $(M=3,50, S D=$ $1,10, A=56,8 \%, N=44)$. Dabei sieht die Mehrheit der Expertinnen und Experten diese Entwickelung in einem mittelfristigen Zeitraum von fünf bis zehn Jahren als realistisch an (siehe Abbildung 44).

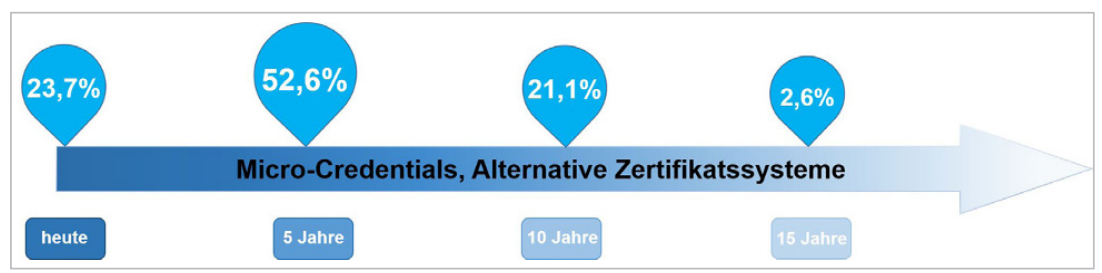

Abb. 44 Geschätzte Zeitdauer für die zunehmende Wichtigkeit von Microcredentials, und alternativen Zertifikatssystemen $(\mathrm{N}=38)$

Dabei ergibt sich ein weiterer Punkt: Die Reputation der Hochschule bestimmt heute den Wert des Abschlusses auf dem Arbeitsmarkt. Studierende, die an einer besonders renommierten Hochschule studiert haben, haben hierbei einen Vorteil gegenüber Studierenden, die an einer Hochschule mit weniger Renommee studiert haben. Über alternative Zertifizierungsmethoden, wie beispielsweise Portfolios, Microcredentials, Badges u. ä. können Studierende zukünftig Zertifizierungen verschiedener Art in ihrem persönlichen Kompetenzportfolio aufbauen. Vielfach sind die dort dokumentierten Fähigkeiten und Erfahrungen auch als qualitative Informationen verfügbar, so dass eine aussagekräftige Informationsbasis entsteht. Diese enthält einerseits akademische Studienleistungen verschiedener Hochschulen und Lehrveranstaltungen als auch weitere Qualifikationen und Kompetenzen, die durch Lernen oder praktische Erfahrungen erworben wurden. Dies wird dazu führen, dass der tatsächliche Praxisbezug des Studiums, die dort gemachten und dokumentierten Erfahrungen und demonstrierbare Handlungskompetenzen zukünftig den Wert des Hochschulabschlusses ausmachen. Immer mehr entwickeln sich sog. Skill-Plattformen im Internet, wie beispielsweise das Hamburger Startup Qompetent (https://www.qompetent.com). Für die Rekrutierung von Tech-Spezialistinnen und Spezialisten gewinnen Job-Matching-Plattformen zunehmend an 
Bedeutung, die auf dem amerikanischen Markt bereits in großer Zahl vertreten sind und gezielt auf den Nachweis von technologischen Fähigkeiten zielen. Bei überfachlichen Qualifikationen (unternehmerisches Handeln, Adaptionsfähigkeit, Kollaboration) können Gamification-Elemente die Rekrutierung deutlich verbessern.

Plattformen wie zum Beispiel Portfolium ermöglichen es den Nutzerinnen und Nutzern, mit geringem Aufwand Arbeitsproben auf die Plattform zu laden, etwa Haus- oder Projektarbeiten. Diese Einträge werden anschließend automatisch analysiert und mit Informationen über eingetragene Berufserfahrungen und die hierbei erworbenen Fähigkeiten verknüpft. Durch ein systematisches Matching dieser Millionen von Nutzerprofilen mit veröffentlichten Stellenausschreibungen ergeben sich umfangreiche technische Qualifikationsprofile, auf deren Basis einzelne Kandidaten mit geeigneten Unternehmen in Kontakt gebracht werden können. Vor allem im Bereich der technologischen Fähigkeiten können spezialisierte Skill-Plattformen die Identifikation und Rekrutierung von Expertinnen und Experten erleichtern und verbessern (Ehlers 2018).

Daneben werden auch Plattformen wichtiger, die zwar nicht das oben beschriebene Matching vornehmen, aber dennoch auf den Nachweis von Fähigkeiten fokussiert sind: So beurteilt der Klout Score des gleichnamigen (inzwischen nicht mehr aktiven) Onlinedienstes die Reichweite und Wirkung der Social-Media-Aktivitäten einer Person. Diese Informationen ermöglichen HR-Abteilungen eine erste Einschätzung der Eignung dieser Person für spezifische Jobs mit Bezug zu Social-Media-Marketing. Im Bereich IT hat sich mittlerweile mit GitHub eine Standardplattform für Arbeitsreferenzen etabliert (https://github.com).

\section{2.3.5 Lebenslanges akademisches Lernen}

Wie können Hochschulen zum aktiven Motor für akademische lebensbegleitende Bildung werden? Glaubt man dem beck 'schen Postulat der Risikogesellschaft (Beck 1986), dann stellt kontinuierliche (akademische) Bildung zünftig einen wichtigen Weg der Risikovermeidung dar. Dabei wandelt sich ständige akademische Weiterbildung im Sinne des lebenslangen Lernens von einer Möglichkeit zur Vermeidung von Lebensrisiken zu einem Zwang, von der Option zur Obligation. Damit einher geht auch die Entwicklung der Employability, die nicht mehr als Berufsfähigkeit, also Vorbereitung auf einen Beruf durch ein universitäres Studium, sondern als Beschäftigungsfähigkeit, also auf die Lebensspanne zielt: Vom , lifetime employment zur , lifetime employability. Das Aufweichen traditioneller Biographiemuster im Zuge der Modernisierung gehört mittlerweile zur weit verbreiteten Erfahrung. Biographien sind durch Unterbrechungen und Veränderungen, durch Neuorientierungen 
und Umstellungen gekennzeichnet sind, und ihnen wohnt das permanente Risiko des Abgleitens oder Abstürzens inne (vgl. Beck, Giddens, \& Lash 1996). Für die Qualifizierung bedeutet das: sie ist nie wirklich abgeschlossen. Auch hier besteht ein Druck auf Hochschulen, Bildungsprozesse verstärkt als episodisch und nicht als einmal und für immer abgeschlossen zu betrachten.

Lebenslanges höheres Lernen befindet sich - so die Meinung des Gremiums der Expertinnen und Experten - auf dem steigenden Ast. Knapp ein Drittel (28\%) gab an, dass lebenslanges höheres Lernen bereits jetzt in einigen Hochschulen als ebenso wichtig wie die akademischen Standardabschlüsse angesehen würde. Vier von zehn Befragten glaubten dieser Trend würde erst in den nächsten fünf Jahren relevant werden (38\%); ca. ein Viertel sprach sich dafür aus, dass dies erst in einem Zehnjahreszeitraum wichtig werden würde (siehe Abbildung 45). Da es sich hierbei jedoch um einen systemischen Wandel handelt, erscheint ein Fünfjahreszeitraum für Wandelunternehmungen sowohl in Hochschulen als auch im Hinblick auf nötige Gesetzesveränderungen reichlich kurz. Jedoch gibt es auf nationaler sowie auf europäischer Ebene bereits durch Programme entwickelte Richtlinien, die den Fokus in Bildungssystemen sehr wohl innerhalb eines Fünf- bis Zehnjahreszeitraums auf lebenslanges höheres Lernen lenken könnten. Speziell unter den Bedingungen schnellen Wissenswandels, Technologieakzeptanzraten und dem sich rasant wandelnden Arbeitskontext, könnte sich dieser Zeitrahmen sogar noch verkürzen.

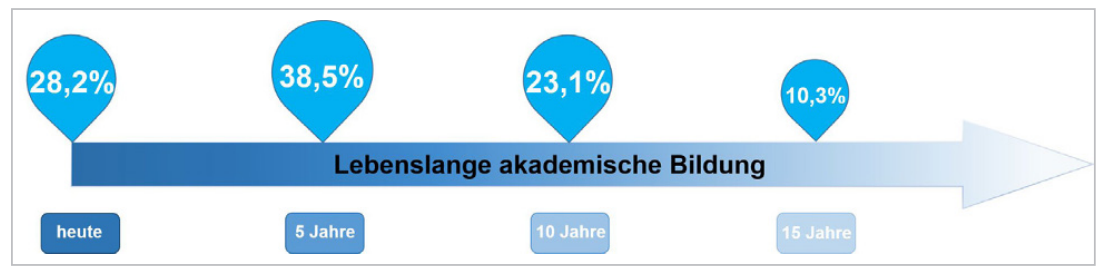

Abb. 45 Geschätzte Zeitdauer für die zunehmende Wichtigkeit lebenslanger akademischer Bildung $(\mathrm{N}=39)$

Das Studium der Zukunft wird der Tatsache Rechnung tragen müssen, dass die stärkere Flexibilisierung am Arbeitsmarkt einen großen Bedarf lebenslanger, akademischer Bildung mit sich bringt. Über 90 Prozent der Befragten sehen innerhalb der nächsten zehn Jahre eine steigende Relevanz episodischer, lebensbegleitender akademischer Bildung, in der bereits erbrachte Leistungen und vorhandene Kompetenzen anerkannt werden (siehe Abbildung 46, $M=3,59, S D=0,96, A=59,1 \%$, $N=44)$ 


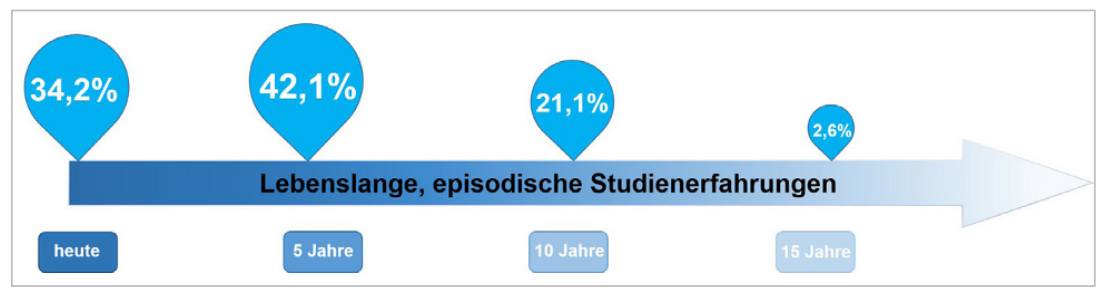

Abb. 46 Geschätzte Zeitdauer für die zunehmende Wichtigkeit lebenslanger, episodischer Studienerfahrungen $(\mathrm{N}=38)$

Die wissensbasierte moderne Gesellschaft impliziert, dass lebenslanges Lernen (LLL) zu einer Notwendigkeit für alle Bevölkerungsteile wird. Lebenslangen Lernens (LLL) beinhaltet „alles Lernen während des gesamten Lebens, das der Verbesserung von Wissen, Qualifikationen und Kompetenzen dient" (Europäische Kommission 2001: 34). Die demografische Entwicklung hat zur Folge, dass alle Menschen im erwerbsfähigen Alter wiederkehrende Bildungsphasen durchlaufen müssen, um das erforderliche hohe Qualifikationsniveau aufrechtzuerhalten. Vor dem Hintergrund der internationalen Migrationsströme müssen auch bei der Entwicklung von Konzepten des LLL auch die Bedürfnisse unterschiedlicher Kulturen mit berücksichtig werden. Die Entwicklung hin zu einer wissensbasierten Wirtschaft stellt zudem immer komplexere Anforderungen an die Arbeitskräfte.

- Während das Studium heute noch weitgehend durch Modul- und Prüfungspläne im Rahmen einer Studienordnung strukturiert ist und nur eine geringe zeitliche Flexibilität und oft auch nur eine bestimmte Bandbreite inhaltlicher Flexibilität vorsieht, wird das Studium der Zukunft durch einen flexiblen Studienverlauf mit großen Wahlbereichen bestimmt werden.

- Während das Studium heute noch stark anhand von Zeiteinheiten strukturiert ist (ECTS), wird das Studium der Zukunft stärker anhand von inhaltlichen Kriterien strukturiert werden.

- Während es heute eine klare Unterscheidung von Teilzeit und Vollzeitstruktur für ein Studium gibt, wird es zukünftig eine flexiblere, individuelle Zeitstruktur geben, und auch verstärkt berufs- und lebensbegleitende Modelle geben.

Insgesamt zeichnet sich ab, dass sich die derzeitige Leitstruktur des Hochschulstudiums sich wandeln wird, wenn sie den Anforderungen an lebensbegleitende Weiterbildung gerecht werden will. Diese wird eine solche Relevanz innerhalb der nächsten 5 bis 10 Jahre erlangen, sodass das derzeitige Vorbereitungsmodell der 
Hochschule durch eine Leitkonzeption des Studiums als lebensbegleitende Konzeption abgelöst werden wird. Dabei gibt es viele Gestaltungsfragen zu beantworten.

Aus wirtschaftspolitischer Perspektive betrachtet, ist eine der Kernaufgaben die Schaffung adäquater Rahmenbedingungen, damit die Beschäftigungsfähigkeit erhalten bleibt bzw. verbessert wird. Aber es geht auch darum, mithilfe des LLL die aktive Teilhabe des Individuums an der Gesellschaft zu verbessern, denn Chancengleichheit und LLL werden als Grundlage der sozialen Inklusion gesehen. Besonders wichtig ist in diesem Zusammenhang die Förderung sozial oder auch geografisch benachteiligter Gruppen sowie von Personen mit niedrigen Basisqualifikationen. Ziel ist es, eine integrative Gesellschaft zu schaffen, die allen Menschen gleiche Zugangschancen zum Lernen und analoge Möglichkeiten der Teilnahme an akademischen Lernangeboten bietet.

Die demografische Entwicklung hat zur Folge, dass alle Menschen im erwerbsfähigen Alter wiederkehrende Bildungsphasen durchlaufen müssen, um das erforderliche hohe Qualifikationsniveau aufrechtzuerhalten. Vor dem Hintergrund der internationalen Migrationsströme müssen bei der Entwicklung von Konzepten des LLL auch die Bedürfnisse unterschiedlicher Kulturen mit berücksichtig werden. Die Entwicklung hin zu einer wissensbasierten Wirtschaft stellt zudem immer komplexere Anforderungen an die Arbeitskräfte. Hochschulen haben sich dieser Aufgabe in den vergangenen Jahren durch einen verhaltenen Aufbau an Kapazitäten im Bereich der wissenschaftlichen Weiterbildung gestellt. Jedoch zeichnet sich ab, dass dies nicht ausreicht.

Es geht bei der zukünftigen Relevanz lebensbegleitender akademischer Bildung nicht (nur) um die Teilnahme an einer Weiterbildung, um Beschäftigungsfähigkeit dort wiederherzustellen wo sie abhandengekommen ist oder lediglich aufrechtzuerhalten. Es geht vielmehr darum, einen Paradigmenwechsel in der akademischen Bildung vorzunehmen, der Hochschulbildung nicht mehr als Vorbereitungsmodell versteht, sondern der Hochschulbildung als ständig andauernde Bildungsaktivität auf Hochschulbildungsniveau gestaltet. Sowohl die organisatorischen Voraussetzungen für die Ausgestaltung eines solchen neuen Paradigmas sind an Hochschulen nicht vorhanden und auch gesetzlich derzeit nicht abbildbar als auch die pädagogisch und kapazitären Voraussetzungen. In Anlehnung an Jochen Robes (2016) könnte eine Liste an Eckpunkten für eine zukünftige Hochschule lebensbegleitender akademischer Bildung wie folgt aussehen:

1. Hochschulen nutzen alle Chancen der Digitalisierung, die sich in Verwaltung, Lehre und Forschung bieten. Lehre und Lernen finden nicht nur im Hörsaal oder Seminarraum statt, sondern schöpfen die Möglichkeiten aus, die das Netz 
heute bietet, um zu informieren, zu diskutieren, zu publizieren und zusammenzuarbeiten.

2. Die LLL-Hochschule ist durchlässiger: Sie ist eine öffentliche Hochschule, die dank innovativer Netztechnologien interessierte Bürgerinnen und Bürger, Arbeitgebende sowie andere Hochschulen, andere Bildungsinstitutionen und Lehrende in ihre eigenen Lehr- und Lernangebote sowie Forschungsprojekte einbindet. Massive Open Online Courses (MOOCs) und Open Educational Resources (OER) sind Teil dieser Entwicklung.

3. Die LLL-Hochschule stellt die Vermittlung des persönlichen Wissensmanagements als Kernkompetenz des LLL in den Mittelpunkt akademischer Bildung. Persönliches Wissensmanagement, das heißt: Informationen finden, bewerten und einordnen, selbst neue Inhalte entwerfen, Informationen und Ergebnisse weitergeben und schließlich sich vernetzen, nicht nur, aber vor allem im virtuellen Raum.

4. Die LLL-Hochschule ist nicht nur in einer bestimmten, zeitlich befristeten Phase der Ausbildung der Dreh- und Angelpunkt der Interessen Studierender, sondern sie begleitet auch nach Beendigung eines Erststudiums weiter. Als Bildungspartner, als Netzwerk, als Informationsressource.

5. Die LLL-Hochschule ist eine Organisation, die auf jeder Ebene - von der Hochschulleitung bis zu den einzelnen Lehrstühlen und Lehrenden - die Idee der Vernetzung aktiv vorantreibt. Sie öffnet Studierenden, Lehrenden, Forschenden und allen Interessierten Räume, um sich auszutauschen; bevor nur noch Google, Apple und LinkedIn den Takt vorgeben. ${ }^{44}$

\section{2.3.6 Verzahnung und Integration von Praxiserfahrungen}

Wie kann die Hochschule der Zukunft digitale Medien nutzen, um das Studium an verschiedenen Orten, arbeitsplatznahes Lernen und praxisintegriertes Studieren zu ermöglichen? Bedingt durch mehr lebensbegleitende Bildung wird auch die berufsbegleitende und arbeitsplatznahe akademische Bildung einen höheren Stellenwert erhalten. Insgesamt ist davon auszugehen, dass arbeitsplatznahe akademische Bildung an Relevanz gewinnt und eine wesentlichen Strukturmerkmal zukünftiger Hochschulangebote darstellt.

44 LinkedIn hat mit dem Erwerb der Skill-Analyse Plattform bright.com sowie dem Internetvideo-Lernportal Lynda und der Entwicklung des sog. „StudyPath Explorer“ bereits heute eine Möglichkeit geschaffen, wie LinkedIn Mitglieder lebenslange und passgenaue Qualifikationen für ihre jeweiligen Ziele erhalten können. 
In praxisintegrierten, praxisnahen oder dualen Studiengängen können digitale Medien genutzt werden, um die dann in der Regel vorhandenen zwei Lernorte - den Lernort Arbeitsplatz und den Lernort Hochschule - miteinander zu vernetzen. Dabei eignen sich beispielsweise Kursformate, die es den Studierenden ermöglichen, auf Inhalte, die sie für Projekt- und Forschungsarbeiten, die am Praxislernort angefertigt werden, zuzugreifen, oder auch das Konzept des reflexiven Schreibens von Lerntagebüchern für Explorations- und Reflexionsaufgaben, die die Studierenden während der Studienphase an der Hochschule anhand von theoretischen Konzepten erarbeiten, und die am Lernort Praxis durchgeführt werden sollen.

\section{C2.3.7 Von der Abschottung zur Durchlässigkeit}

Wie können Hochschulen sich noch mehr für alternative Zielgruppen öffnen und Anschlussfähigkeit und Durchlässigkeit mit verschiedenen Bildungssektoren erhöhen? Hochschulbildungssysteme werden durch alternativen Studienverläufe zunehmend offen und durchlässig - so formulieren es die befragten Expertinnen und Experten der internationalen Delphi-Befragung mit großer Übereinstimmung $(\mathrm{M}=3,95, \mathrm{SD}=$ $0,82, \mathrm{~A}=81,8 \%, \mathrm{~N}=44$ ). Die bislang existierenden und geltenden Grenzen zwischen Schule, Berufsausbildung und Hochschule werden zukünftig stärker verschwimmen und eine erhöhte Durchlässigkeit wird von hoher Bedeutung sein.

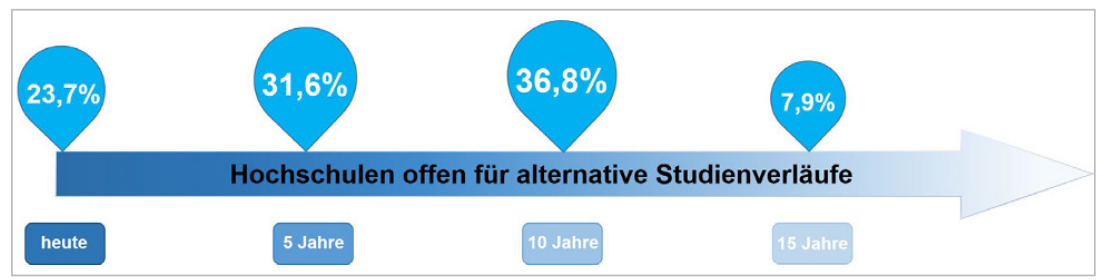

Abb. 47 Geschätzte Zeitdauer für die zunehmende Wichtigkeit für die Offenheit von Hochschulen für alternative Studienverläufe $(\mathrm{N}=38)$

Die befragten Expertinnen und Experten schätzen den Zeitraum, in dem diese Entwicklung realisiert wird zwischen fünf und zehn Jahren ein (siehe Abbildung 47). Ziel wird es dabei sein, ein durchlässiges Kontinuum zwischen den Bildungsbereichen Schule, Berufsausbildung und Hochschule und den jeweiligen anschlussfähigen Bildungsniveaus der nationalen und Europäischen Qualifikationsrahmen 
zu schaffen. Diese Entwicklung wird insbesondere durch die Notwendigkeit verstärkter lebensbegleitender Bildung gefördert werden. Denn neben der Steigerung der individuellen Lernbereitschaft gilt dabei das Ziel, die mehr oder minder bestehende Versäulung des Bildungssystems aufzulösen, um die Flexibilisierung der individuellen Lernbiographien zu ermöglichen (Bohlinger \& Heidecke 2009: 454). Die Forderung nach systemübergreifenden und durchlässigen Lernwegen steht im Widerspruch zu einem hochgradig segmentierten Bildungssystem, was den Individuen oftmals eine endgültige und schwerlich zu korrigierender Entscheidung über eine beruflich-betriebliche oder akademische Qualifizierungskarriere abverlangt.

Die bislang tradierten institutionalisierten Abschottungsmechanismen zwischen Berufsbildungs- und Hochschulsystem bergen für die Zukunft Probleme, denn nach einschlägigen Prognosen wird ein Mangel an akademisch qualifizierten Fachkräften erwartet (Nickel \& Leusing 2009: 19), der die Relevanz des Lernortes Hochschule explizit unterstreicht. Da nicht alle diejenigen, die zukünftig studieren wollen auch eine allgemeine oder gebundene Hochschulzugangsberechtigung besitzen, steht auch das Thema der Etablierung flexiblerer Übergangspassagen zwischen Berufs- und Hochschulbildung, und zwar jenseits formaler Berechtigungen, ganz oben auf der politischen Agenda (KMK 2009a / 2009b). Die Realisierung durchlässiger und lebenslanger Lernmöglichkeiten in der Hochschule vollzieht sich auf unterschiedlichen Gestaltungsebenen - ausgehend von einer Erweiterung auf verschiedene Zielgruppen in Verbindung mit bildungspolitischen Perspektiven, um eine Verbreiterung der Zugangswege auch für nicht-traditionelle Studierende mittels Anrechnungsverfahren zu ermöglichen.

Die Öffnung der Hochschulen für immer mehr junge Menschen eines Jahrgangs führt automatisch dazu, dass auch nicht-traditionelle Zielgruppen an die Hochschule strömen und erhöht die Diversität der Lebenslagen, in denen Menschen sich akademischer Bildung zuwenden. Das stellt für Hochschulen eine Herausforderung in Bezug auf den Studienerfolg dar. Gerade in den mathematisch naturwissenschaftlichen Studienfächern sind Hochschulen häufig mit mathematischen Eingangsqualifikationen der Studienanfängerinnen und Studienanfänger konfrontiert, die nicht ausreichen, um die Studieneingangsphase erfolgreich zu überstehen (Heublein et al. 2014). Mehr und mehr Hochschulen experimentieren nun mit Onlinekursen, die die Studierenden bereits im Vorfeld zum Studium belegen und die ihnen die Möglichkeit geben, sich in den notwendigen Bereichen das entsprechende Qualifikationsniveau zu erarbeiten. ${ }^{45}$

45 Das EU Projekt „OER Test“ hat die hierbei denkbaren Möglichkeiten aufgearbeitet und publiziert: https://oerknowledgecloud.org/sites/oerknowledgecloud.org/files/Open-Learning-Recognition.pdf. 
Eine weitere Möglichkeit, eher zur Unterstützung der Öffnung von Hochschulen auch für nicht-traditionelle Zielgruppen, sind sogenannte Brückenkurse, die mit Äquivalenzprüfungen gekoppelt werden können. Wollen beispielsweise Berufstätige mit langer Berufspraxis wieder ins Studium zurück, bietet es sich an, sie mit Onboarding Kursen, Propädeutika oder ähnlich zu unterstützen, um wieder in ein akademisches Studium hineinzukommen - und zwar ohne, dass sie regelmäßig bereits in Präsenzkurse an die Hochschule kommen müssen.

\section{C2.4 Zusammenfassung: Die Zukunft der Hochschulen kommt schneller als gedacht}

Viele Universitäten haben sich bereits auf den Weg in die Zukunft gemacht. Dabei werden die im Beitrag beschriebenen zwei Haupteinflussfaktoren, die Digitalisierung und die zunehmende Bedeutung von akademischer Qualifikation als normalbiographische Erfahrung, so wirken, dass akademische Bildung sich in Organisation, Didaktik, Profil und institutioneller Ausrichtung weiterentwickeln muss. Digitalisierung ist dabei kein Allheilmittel für Hochschulen und die hochschuldidaktische Gestaltung. Aber Hochschulbildung digital hat das Potenzial die Transformation der Hochschule in Bezug auf ihre gesellschaftlichen Anforderungen zu unterstützen. Hochschulen stehen daher heute mehr denn je vor einer Gestaltungsaufgabe, digitalen Medien sinnvoll zu integrieren.

Hochschulen sind zu Reallaboren geworden, in denen mutige Konzeptionen entwickelt werden. Digitalisierung wird dabei meistens als Didaktisierung erlebt, denn mit dem Einsatz von neuen Medien stellen sich lernorganisatorische und didaktische Fragen (endlich) wieder neu. Es sind digitale Medien, die oft zunächst erst sichtbar machen, dass das Ideal der Universitas im Hochschulalltag nicht gelebt wird. Die NextSkills Initiative bietet konkrete Ansatzpunkte für die Entwicklung der Hochschule der Zukunft (siehe Abbildung 48).

Zusätzlich bieten immer mehr Hochschulen ihren Studierenden an, sie in der Studieneingangsphase bei der Studienorganisation und ihren mathematischen Kompetenzen zu unterstützen: http://www.optes.de 


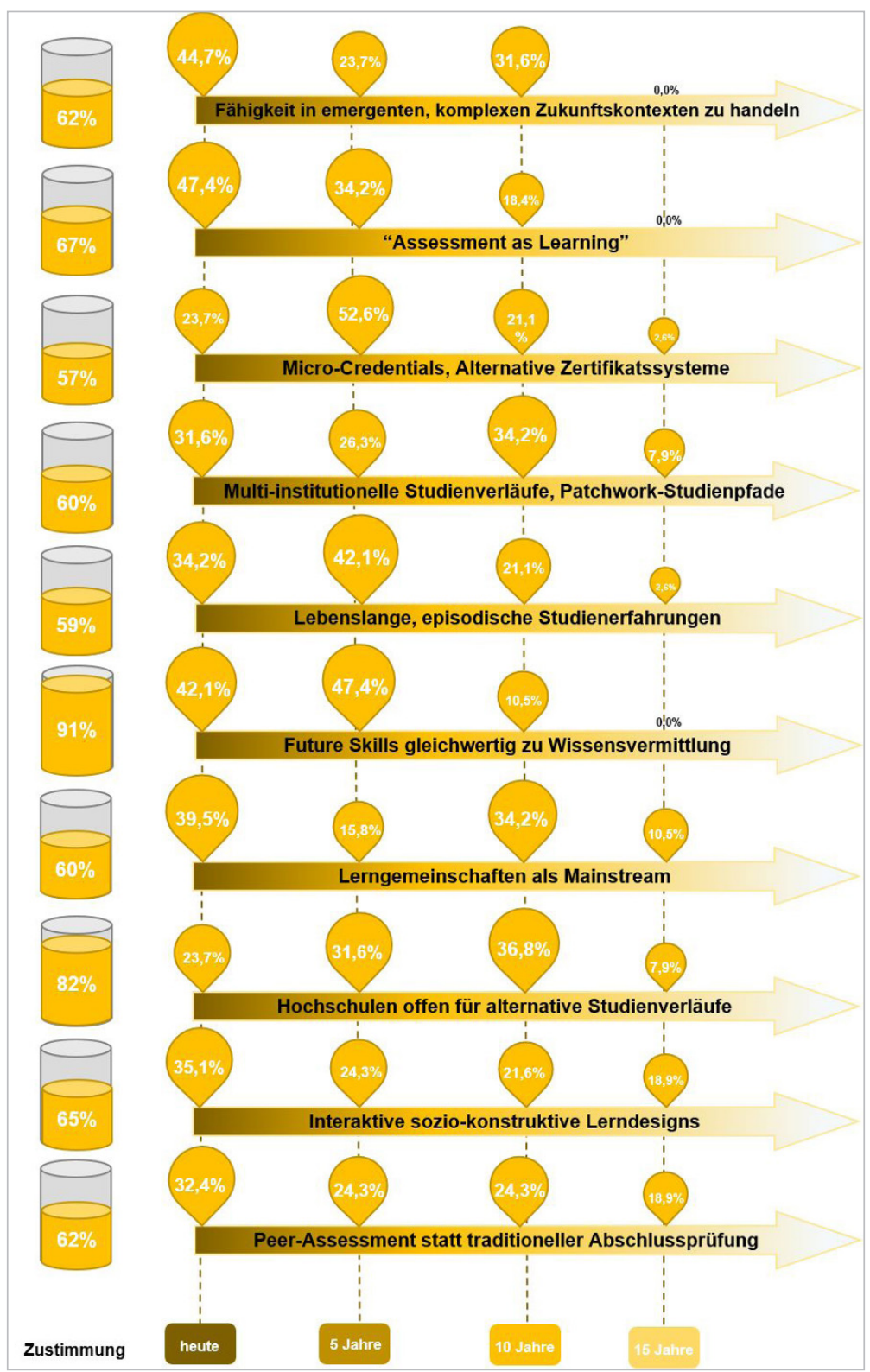

Abb. 48 Entwicklungsgeschwindigkeit für ausgewählte Hochschulentwicklungen 
Insgesamt drückte das internationale Expert(inn)ensample seine Zustimmung zu den obigen Aussagen aus (alle Mittelwerte bewegten sich zwischen 3,54 und 4,19). Am wenigsten Zustimmung ergab sich für die Aussage, Microcredentials würden die traditionellen Formen der Zertifizierung eines gesamten Studienabschlusses ersetzen $(M=3,54, S D=1,10)$. Dagegen wurden folgende Elemente als charakteristische Faktoren für die Zukunft der Hochschulbildung eingeschätzt: erstens, die Wichtigkeit von Future Skills $(M=4,19, S D=0,71)$ sowie zweitens, eine damit einhergehende und notwendige Anpassung entsprechender Qualitätsstandards um sicherzustellen, dass die Employability der Studierenden weiterhin gewährleistet bliebe $(M=4,15, S D=0,82)$ und drittens, ein erhöhtes Maß an Diversität und Permeabilität im Hinblick auf die Hochschulen, wodurch sich der Weg für alternative Studienverläufe und non-traditionale Studierendengruppen ebnen würde ( $M=$ $4,00, S D=0,84)$. Um Future Skills in Hochschulen ausbilden zu können, verwiesen die Befragten des Delphis auf die Wichtigkeit, Lehrpersonal entsprechend auszubilden. Nur wenn Lehrende dazu in der Lage sind, die richtigen Lehrmethoden einzusetzen, sei es nach Expert(inn)enmeinung möglich, diese Skills auch bei den Studierenden auszubilden. Obwohl die Expertinnen und Experten die Rolle der Qualitätsstandards als Treiber des Wandels kritisierten, stimmten sie dennoch generell zu, dass es nötig sei, diese im Hinblick auf die neuen Anforderungen für Studierende entsprechend anzupassen. Die zunehmende Offenheit der Hochschulen wurde als gewinnbringend für die ganze Gesellschaft eingeschätzt; jedoch äußerten die befragten Expertinnen und Experten auch hier Bedenken, inwieweit Hochschulen schon in der Lage seien, sich einem solchen Wandel zu unterziehen und ob die Veränderungen tatsächlich das Potential hätten, soziale Differenzen auszugleichen oder diese zumindest zu verringern.

Darüber hinaus identifizierten die Expertinnen und Experten fünf Faktoren, welche sie als bereits jetzt schon hoch relevant für die Organisation der Hochschulbildung erachteten ${ }^{46}$ Hierbei handelte es sich um die Bewertung des Lernens um des Lernens Willen (formative Bewertung), das institutionelle Hochschul-Patchwork, Peer-Evaluation und -Validierung sowie alternative Studienverläufe.

Dem internationalen Expert(inn)ensample zufolge, werden voraussichtlich bereits in den nächsten fünf Jahren vor allem zwei Tendenzen die Funktionsweise von Hochschulen verändern: ein gewandeltes Verständnis von Hochschulen als transfer-orientierten Orten anstelle deren momentanem Fokus auf Fachwissensvermittlung sowie auf micro-credentialing beruhenden Qualifikationsnachweisen. Auch die Fähigkeit von Studierenden, sich in komplexen, unbekannten Zukunfts-

46 „Already relevant“ erhielt die meisten Stimmen für diesen Faktor, wurde hierfür als Bemessungsgrundlage verwendet. 
kontexten zurechtfinden zu können als richtungsweisender Faktor für künftige Hochschulbildung wird - so die Meinung der Expertinnen und Experten - bereits auf kurze Sicht (in den nächsten fünf Jahren) relevant werden.

Als mittelfristig relevant werdend identifizierte das Sample den Wandel hin zu sozio-konstruktiven Ansätzen für Hochschullernen und einen fluideren Weg der Studierenden zwischen einzelnen Institutionen während ihres Studienverlaufs. Letzteres wird durch die Anerkennung von bereits erbrachten Studienleistungen und vorhandenen Kompetenzen erleichtert. Obwohl der Sample-Mittelwert darauf hindeutet, dass diese beiden letztgenannten Trends erst in den kommenden zehn Jahren relevant sein werden, weißt die Betrachtung der Sample-Mehrheit in beiden Fällen allerdings darauf hin, dass beide Faktoren bereits heute relevant sind. 